\title{
SYNTHESIS, CHARACTERIZATION AND ANTIMICROBIAL ACTIVITY OF NOVEL PYRAZOLO[3,4-b]PYRIDINES AND THEIR SPIRO- HETEROCYCLIC DERIVATIVES
}

\author{
Shawkat A. Abdelmohsen\#, Talaat I. El-Emary \\ Department of Chemistry, Faculty of Science, Assiut University, Assiut 71516, Egypt \\ E-mail address: shawk662001@yahoo.com
}

\begin{abstract}
The present work describes the synthesis of a novel series of heterocyclic moieties derived from 5-acetylpyrazolo[3,4b]pyridine (1). The formation of chalcones (2a-d) was utilized to synthesize pyrazoline, isoxazoline and pyrimidine derivatives (3-10). Thiosemicarbazone and semicarbazone $(\mathbf{1 1}, \mathbf{1 7})$ were utilized to synthesize other new triazolethiones, thiadiazole and selenadiazole derivatives (11-19). Some new spiro derivatives (22-25) were synthesized by the reaction of chalcone (21) of 1 and isatine with hydrazines, hydroxyl amines and thiourea. Also, The reaction of 1 with cyanoacetyl hydrazine gave the hydrazide-hydrazone derivative 26, which was allowed to react with aromatic aldehydes and $\alpha-$ cyanocinnamonitrile to afford coumarine and substituted pyridine derivatives $(\mathbf{2 8}, \mathbf{2 9})$. The structures of all the new compounds have been established on the basis of their analytical and spectral data. Twenty two of the synthesized compounds were also evaluated for their antibacterial and antifungal activity against various strains of bacteria and fungi and most are found to possess promising antimicrobial activity when compared with Chloramphenicol and Clotrimazole
\end{abstract}

Keywords: Pyrazolo[3,4-b]pyridine; Chalcones; Heterocyles; Spiroindolylpyrazolopyridine; Antimicrobial

\section{Council for Innovative Research}

Peer Review Research Publishing System

Journal: Journal of Advances in Chemistry

Vol. 10, No. 7

editorjaconline@gmail.com

www.cirjac.com 


\section{INTRODUCTION}

The pyrazolo[3,4-b]pyridine moieties represent important building blocks in both natural and synthetic bioactive compounds. ${ }^{1}$ They are attractive targets in organic synthesis due to their significant biological activities such as xanthine oxidase inhibitors, cholesterol formation-inhibitor, and Anti-Alzheimer, ${ }^{2,3}$ analgesic, ${ }^{4}$ anxiolytic, ${ }^{5}$ hypnotic, ${ }^{6}$ antiviral, ${ }^{7}$ anti-HIV, ${ }^{8}$ corticotropin-Releasing Factor (CRF) antagonist, ${ }_{9,10}$ antidiabetic, ${ }_{11}$ antiarrhythmic, ${ }^{12}$ antitumor, 13 antimalarial. ${ }^{14}$ They also show antimicrobial and antiparasitic activities. ${ }^{15-17}$ In addition, they are used as anti-HIV-1, ${ }^{18}$ as a potential glucocorticoid receptor ligand for positron emission tomography (PET). ${ }_{20}$ It was found that some derivatives of pyrazolo[3,4-b]pyridine were belived to be effective as antileishmanial agents, ${ }^{20}$ inhibitors of erectile dysfunctions. ${ }^{21}$ Taking all the above into consideration and in continuation of our previous work directed to synthesis of new heterocycles engaged with pyrazole nuclei, ${ }^{22-25}$ we describe herein the utilization of the 1-(3,6-dimethyl-1-phenyl-1H-pyrazolo[3,4b]pyridine-5-yl)ethanone (1) as the key intermediate in the synthesis of novel pyrazolo[3,4-b]pyridine members along with studies of the effect of some of them as antifungal and as antibacterial agents.

\section{RESULTS AND DISCUSSION}

The starting compound 2a-d were synthesized via the the Claisen-Schmidt reaction of 1-(3,6-dimethyl-1-phenyl-1Hpyrazolo[3,4-b]pyridine-5-yl)ethanone (1) ${ }^{26}$ with different p-substituted aldehydes in ethanol and in presence of aqueous potassium hydroxide $(25 \%)$. Presence of $\alpha$ - $\beta$-unsaturated keto function makes chalcones very prone to undergo reaction with bidentate nucleophiles to give five and six membered heterocyclic moieties. We intended to utilized this procedure to explore the formation of compounds 3-9 containing the pyrazole, isoxazole and pyrimidine rings linked on 5-position of pyrazolo[3,4-b]pyridine nucleus. Thus, cyclocondensation of chalcones $2 c(R=C l)$ with hydrazine hydrate or phenyl hydrazine in dry ethanol gave the corresponding pyrazole and $\mathrm{N}$-phenylpyrazole derivatives $\mathbf{3 a}$ and $\mathbf{3 b}$, respectively. The ${ }^{1} \mathrm{H}$ NMR spectrum of compound $3 \mathbf{a}$ and $\mathbf{3 b}$ showed doublet of doublets of $-\mathrm{CH}_{2}$ near about $\delta 3.12-4.15 \mathrm{ppm}$ and doublet of doublets of $-\mathrm{CH}$ at $\delta 4.85-5.27 \mathrm{ppm}$, confirmed the cyclisation in pyrazoline. Also, the reaction of 2c with hydroxylaminehydrochloride in the presence of anhydrous sodium acetate led to the formation of 5-[5-(4-chlorophenyl)4,5-dihydro-1,2-isoxazol-3-yl]-3,6-dimethyl-1-phenyl-1H-pyrazolo[3,4-b]pyridine (4). The IR spectra of 4 clearly showed the formation of isoxazoline ring by the appearance of band at $1509 \mathrm{~cm}^{-1}$ for $(=\mathrm{C}=\mathrm{N}-\mathrm{O})$. ${ }^{1} \mathrm{H}$ NMR data also confirmed the synthesis by showing a doublet at $\delta 3.75 \mathrm{ppm}$ for $2 \mathrm{H}$ and a triplet at $\delta 4.40 \mathrm{ppm}$ for $1 \mathrm{H}$ of isoxazoline ring. Similarly, the reaction of $\mathbf{2 c}$ with urea and/or thiourea in presence of aqueous potassium hydroxide (10\%) gave the corresponding pyrimidinone and thiopyrimdine derivatives $(\mathbf{5 a}, \mathbf{b})$ respectively. S-alkylated products $(\mathbf{6 a}, \mathbf{b})$ were obtained upon treatment of $\mathbf{5 b}$ with ethyl iodide and benzyl bromide in the presence of anhydrous sodium acetate in refluxing ethanol. Moreover, treatment of $2 c$ with guanidine hydrochloride in dry ethanol containing sodium hydroxide solution(10\%) yielded 2-Amino-4(4-chlorophenyl)-6-(3,6-dimethyl-1-phenyl-1 H-pyrazolo[3,4-b]pyridin-5-yl)pyrimidine (7). Heating of 7 with benzaldehyde in refluxing methanol in the presence of few drops of acetic acid resulted in the formation of the corresponding Schiff base (8). Condensation of chalcones with thiosemicarbazide has became an extremely popular method for making pyrazoline derivatives. Thus, treatment of $\mathbf{2 c}$ with thiosemicarbazide in an ethanolic sodium hydroxide solution,yielded the pyrazole carbothioamide (9). The IR spectra of compound 9 showed intense bands at 3425, 3250, 1580 and $1370 \mathrm{~cm}^{-1} \mathrm{due}$ to $\mathrm{NH}_{2}$, $\mathrm{C}=\mathrm{N}$ and $\mathrm{C}=\mathrm{S}$ groups, respectively. In addition, the absorption band at $1120 \mathrm{~cm}^{-1}$ was attributed to the $\mathrm{C}-\mathrm{N}$, which also confirm the formation of desired pyrazoline ring. In the ${ }^{1}$ HNMR spectra, pyrazoline protons HA and HB are germinal protons at C4 carbon appears at 3.46 and $4.0 \mathrm{ppm}$ as doublet of doublets. The $\mathrm{CH}$ proton also appeared as doublet of doublets at $5.15 \mathrm{ppm}$ due to vicinal coupling with two non-equivalent germinal protons of C4 carbon. Compound 9 underwent ready cyclization upon treatment with $\alpha$-haloketones in the presence of anhydrous sodium acetate to afford 5 (4-Chlorophenyl)-4,5-dihydro-1-(4-substitutedthiazol-2-yl)-1-H-pyrazol-3-yl)-3,6-dimethyl-1-phenyl-1 H-pyrazolo[3,4-

b]pyridines $(\mathbf{1 0 a}, \mathbf{b})$ (Scheme 1). On the other hand, condensation of the target compound (1) in boiling ethanol with thiosemicarbazide afforded the corresponding thiosemicarbazone (11), ${ }^{27}$ which was allowed to react with $\alpha$-haloketones to yield the corresponding thiazolidines derivatives $(\mathbf{1 2 a}, \mathbf{b})$. In addition, the thiosemicarbazone (11) was heated with methanolic $\mathrm{HCl}$ to give 5-methyl-5-(3,6-dimethyl-1-phenyl-1H-pyrazolo[3,4-b]pyridine-5-yl)-1,2,4-triazolidine-3-thione (13). A further support for the structure of (13) was achieved by synthesis of the compound via another route by boiling a solution of $\mathbf{1}$ in methanolic $\mathrm{HCl}$. All of spectral analyses completely identical and confirmed the formation of compound 13 by the two routes. We have observed that extensive thiol-thione tautomerism exists in compound 13. In most cases of nucleophilic substitution of an alkyl halide on a thioamide system, sulfur atom lattack is favored. ${ }^{28}$ Thus, treatment of 13 with 1-bromo-2-methoxyethane in warm DMF in the presence of potassium carbonate afforded the desired products 14 and 15. Column chromatography of the crude material was allowed of the isolation of both isomers. The S-alkylation product 14 as a major (79\% yield) and the other $\mathrm{N}$-alkylation product 15 (14.8\% yield). ${ }^{1} \mathrm{HNMR}$ and ${ }^{13} \mathrm{CNMR}$ spectra of the synthesized compounds provided proof of the $\mathrm{S}$ - and N-alkylated triazoles 14 and 15, respectively. The $\mathrm{CH}_{2}$ protons adjacent to the sulfur atom in compound $\mathbf{1 4}$ appears at $3.75 \mathrm{ppm}$ and the carbon resonates at $33.6 \mathrm{ppm}$. $\mathrm{However}_{2} \mathrm{CH}_{2}$ protons adjacent to the nitrogen atom in compound 15 are more download-shifted to 4.60 and the carbon resonates at $46.27 \mathrm{ppm}$. Finally, the ${ }^{13} \mathrm{CNMR}$ spectra show peak at $172 \mathrm{ppm}$ corresponding to the $\mathrm{C}=\mathrm{S}$ group associated with the $\mathrm{N}$ - 


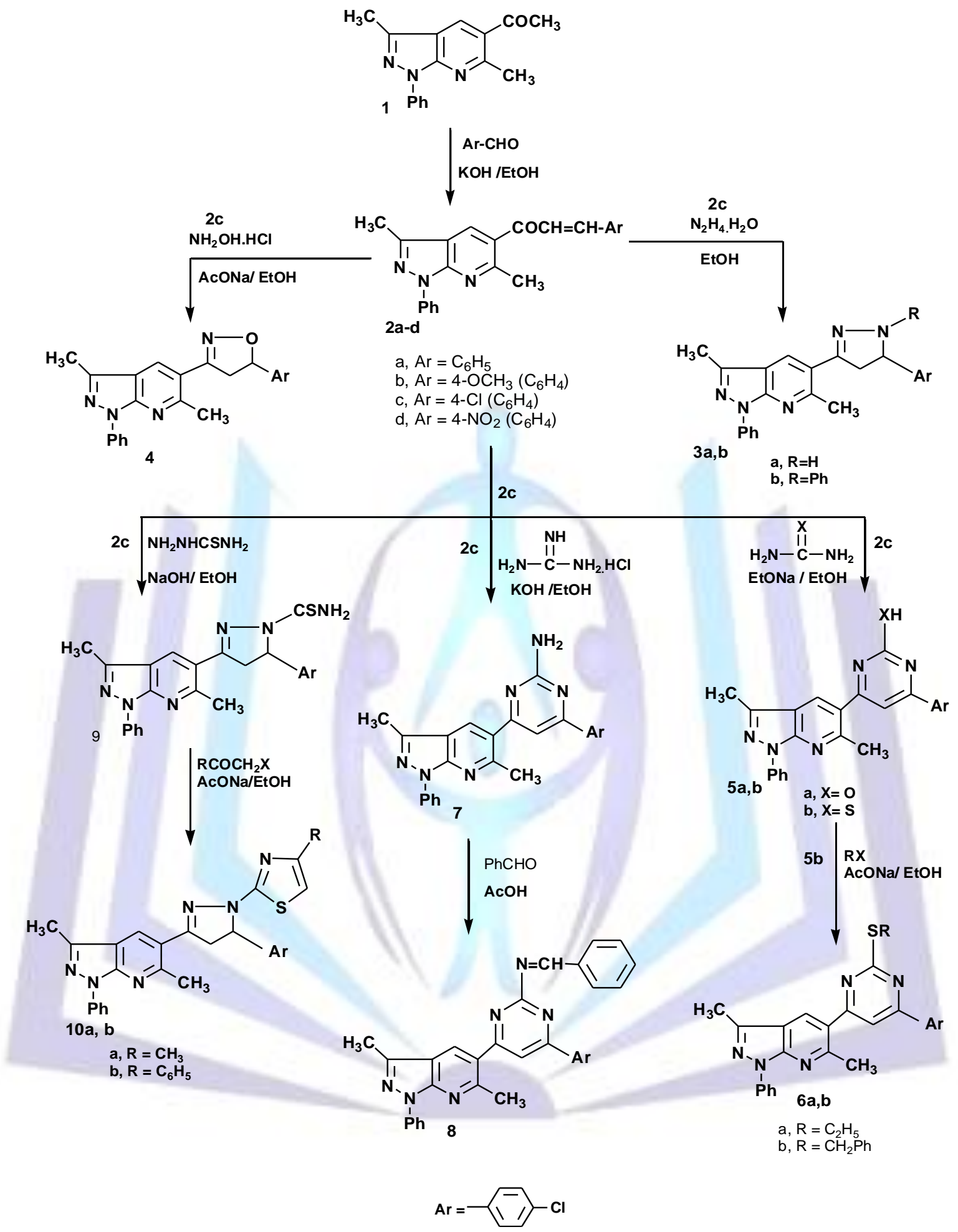

Scheme 1 Pathway for synthesis of compounds 2-10

alkylated derivative 15, whereas the S-alkylated derivative 14 gave peak at $152 \mathrm{ppm}$ for the same carbon corresponding to $\mathrm{C}$-S group. Also, compound 13 was allowed to react with $\mathrm{N}$-phenyl maleimide in acetic acid to afford 2-[2-(3,6-dimethyl1-phenyl-1H-pyrazolo[3,4-b]pyridine-5-yl)-2-methyl-6-oxo-1,2,5,6-tetrahydro-[1,3]thiazolo[3,2-b][1,2,4]triazol-5-yl]-Nphenyl-cetamide (16). The IR spectra of thiazolotriazole (16) showed the absorption bands at 1710 and $1585 \mathrm{~cm}^{-1}$ corresponding to $\mathrm{C}=\mathrm{O}$ and $\mathrm{C}=\mathrm{N}$, respectively. In the ${ }^{1} \mathrm{H}$ NMR spectra of compound 16, protons of $\mathrm{CH}_{2}-\mathrm{CH}_{\text {fragment }}$ showed the characteristic patern of an ABX system. The chemical shifts of the protons $H A, H B$ and $H X$ are doublet of doublets at $2.8,3.3$ and at $4.6 \mathrm{ppm}$, respectively. The acetamide $\mathrm{NH}$ proton appeared at a sharp singlet at $13.30 \mathrm{ppm}$. In addition, the ${ }^{13} \mathrm{C}$ CNMR spectra displayed characteristic two singlets at 173.25 and $178.85 \mathrm{ppm}$ due to the acetamide carbamoyl carbon $(\mathrm{CONH})$ and the thiazolidinone carbonyl carbon $(\mathrm{CO}-\mathrm{N}-)$, respectively. Condensation of the target 
compound 4-acetyl-3-methyl-5-oxo-1-phenyl-2-pyrazoline (1) in boiling ethanol with semicarbazide hydrochloride afforded the corresponding semicarbazone (17). When the semicarbazone 17 was reacted with thionyl chloride it gave 4-(3-methyl-5-oxo-1-phenyl-2-pyrazolin-4-yl)-1,2,3-thiadiazole (18). On the other hand, treatment of 17 with selenium dioxide, 4-(3-methyl-5-oxo-1-phenyl-2-pyrazolin-4-yl)-1,2,3-selenadiazole (19) was obtained. Structure of compounds 18 and 19 was supported in the basis of elemental and spectral analysis. FT-IR spectra exhibited the absence of (NH) and $\left(\mathrm{NH}_{2}\right)$ absorption bands of the semicarbazone and revealed a characteristic absorption band at $820 \mathrm{~cm}^{-1}$ due to CSe-N. ${ }^{29}$ HNMR spectra also appeared two singlets at $\delta 9.75$ and $10.12 \mathrm{ppm}$ corresponding to the $\mathrm{CH}$ protons of thiaziazole and selenadiazole, respectively (Scheme 2).

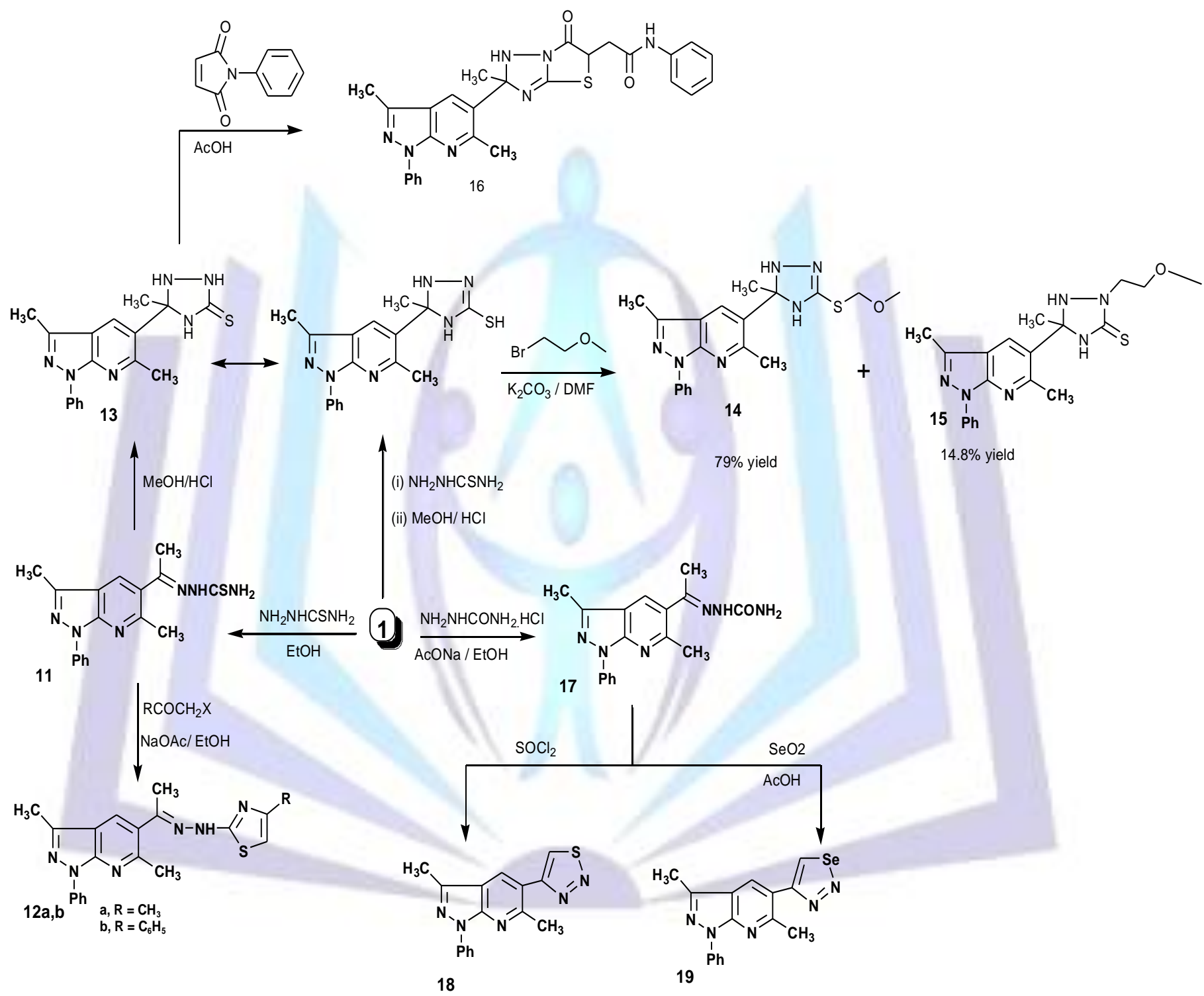

Scheme 2 Pathway for synthesis of compounds 11-19

Crossed aldol condensation between 5-acetylpyrazolo[3,4-b]pyridine (1) and isatin was carried out in the presence of diethyl amine as a basic catalyst gave rise the formation of 3-hydroxy-3-(2-(3,6-Dimethyl-1-phenyl-1H-pyrazolo[3,4b]pyridine-5-yl)-2-oxoethyl)indolin-2-one (20). Dehydration of 20 using Ethanol-HCl mixture afforded the corresponding chalcone 21. Nucleophilic Michael addition of hydrazine hydrate to the chalcone $\mathbf{2 1}$ gave spiropyrazoline derivative (22) (method A). Moreover, spiropyrazoline (22) was obtained in an excellent yield via a one-pot synthesis without the isolation of the intermediate 21. Thus, compound 1 reacted with isatin in the presence of diethylamine for $30 \mathrm{~min}$ at room temperature and the formed solid was treated with glacial acetic acid and hydrochloric acid for another $30 \mathrm{~min}$ at $80 \mathrm{C}$. Hydrazine hydrate was then added to the previous acidic solution to give the spiropyrazoline (22) (method B). The chalcone derivative (21) when interacted with phenylhdyrazine in ethanol as solvent, gave the required spiro $\mathrm{N}$ - 
phenylpyrazoline derivative (23). 3'-(3,6-dimethyl-1-phenyl-1H-pyrazolo[3,4-b]pyridine-5-yl)-4'-dihydrospiro[indol-3,5'isoxazole]-2- $(1 \mathrm{H})$-one $(\mathbf{2 4})$ was obtained by the refluxing of the chalcone $\mathbf{2 1}$ with hydroxylamine hydrochloride in ethanol in the presence of sodium acetate as catalyst. Also, compound 21 was interacted with thiourea in ethanol as solvent and in the presence of potassium hydroxide, gave the 6'-(3,6-dimethyl-1-phenyl-1H-pyrazolo[3,4-b]pyridine-5-yl)- spiro[indol-3,4' pyrimidin-2'- $(1 \mathrm{H})$ thione]-2-(1H)-one (25) (Scheme 3).

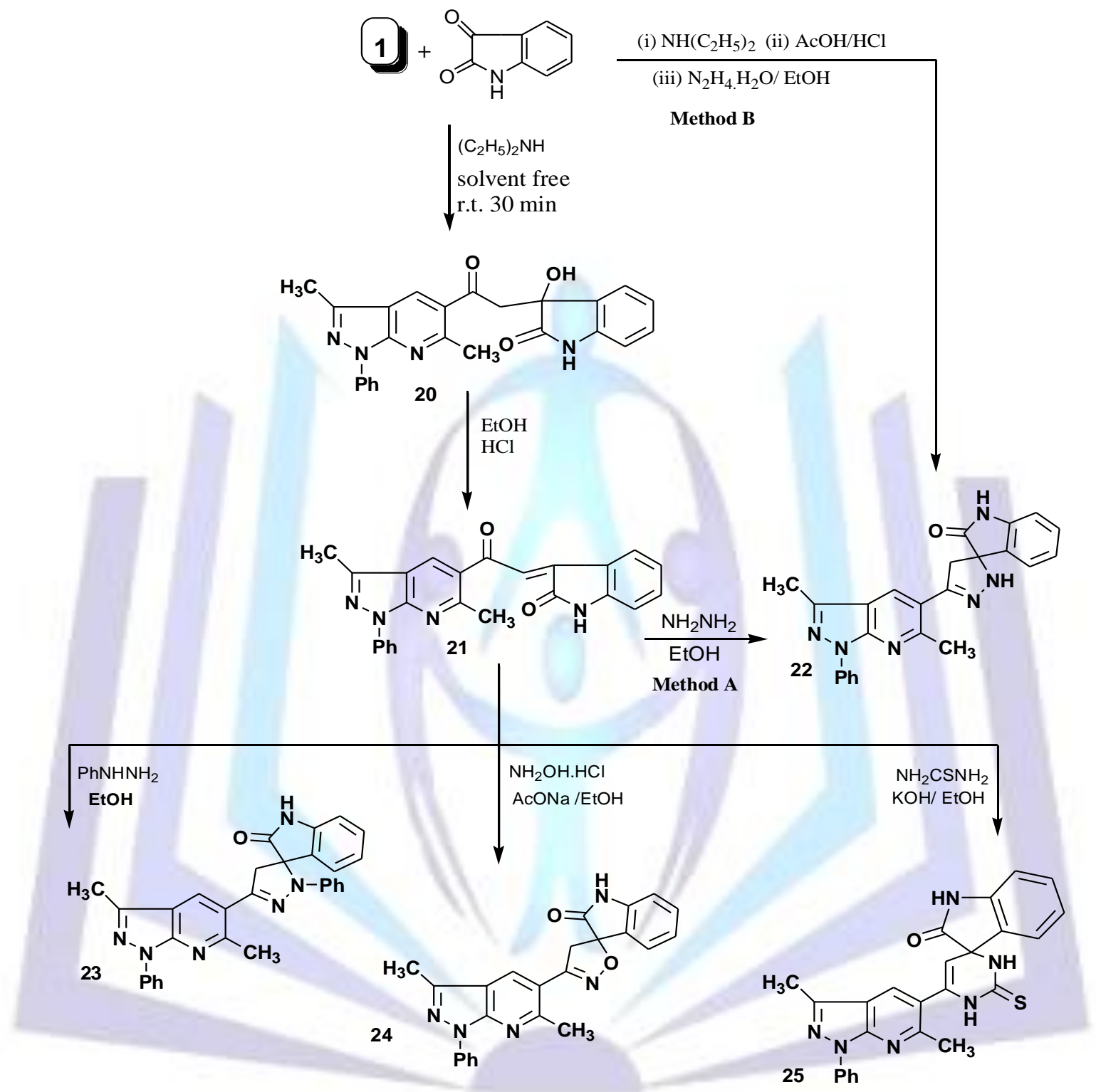

Scheme 3 Pathway for synthesis of compounds 20-25

${ }^{1}$ HNMR spectra confirmed the formation of the chalcone (21) by the absence of a peak at $\delta 3.15$ ppm corresponding to $\mathrm{CH}_{2}$ protons in the aldol product (20). ${ }^{1} \mathrm{HNMR}$ spectra of the spiropyrazoline (22) revealed the presence of the characteristic peaks at $\delta 3.65,6.30$ and 8.63 ppm due to $\mathrm{CH}_{2}$ protons of pyrazoline and $\mathrm{NH}$ protons of each pyrazoline and indoline moieties, respectively. The structure of $\mathbf{2 5}$ was indicated by spectral methods, its IR spectra revealed characteristic bands at $3340,3210,3180$ and $1180 \mathrm{~cm}^{-1}$ corresponding to $(\mathrm{NH})$ and $(\mathrm{C}=\mathrm{S})$, respectively. The $1 \mathrm{HNMR}$ spectra of 25 showed the presence of two broad exchangeable singlets at $\delta 8.85$ and $9.90 \mathrm{ppm}$ for two $\mathrm{NH}$ protons of pyrimidine ring. With the aim of obtaining novel hydrazide-hydrazone with a wide spectrum of pharmaceutical applications, ${ }^{30}$ we report herein the reaction of 5-acetylpyrazolo[3,4-b]pyridine (1) with cyanoacetyl hydrazine in dioxane to yield the corresponding acetohydrazide-hydrazine derivative (26). The structure of compound 26 was confirmed based on its analytical and spectral data. Thus, the $1 \mathrm{H}-\mathrm{NMR}$ showed a singlet at $\delta 4.40$ for the $\mathrm{CH}_{2}$ group and a singlet $\left(\mathrm{D}_{2} \mathrm{O}\right.$ exchangeable) at $\delta 10.95$ for the $\mathrm{NH}$ group. Moreover, the ${ }^{13} \mathrm{C}$ - NMR spectrum showed peaks at $\delta 12.90,26.80,113.64$ and $174.19 \mathrm{ppm}$ corresponding to $\left(\mathrm{CH}_{3}\right),\left(\mathrm{CH}_{2}\right),(\mathrm{CN})$ and $(\mathrm{C}=\mathrm{O})$, resoectively. Further structure elucidation of compound 26 was obtained through the study of its reactivity towards chemical reagents. Thus, reaction of 26 with $p$ chlorobenzaldehyde gave the corresponding benzal derivative (27). On the other hand, the coumarin derivative (28) was 
obtained via the reaction of $\mathbf{2 6}$ with salicyladehyde . Finally, the reaction of $\mathbf{2 6}$ with $\alpha$-cyanocinnamonitrile gave 6-amino-1[1-(3,6- dimethyl-1-phenyl-1H-pyrazolo[3,4-b]pyridine-5-yl)ethylideneamino]-2-oxo-4-phenyl-1,2-dihydropyridine-3,5dicarbonitrile (29). The structure of 29 was confirmed by spectral and analytical data. Thus, The IR spectra revealed the presence of absorption bands at 3452, 3368, 2229 and $2189 \mathrm{~cm}^{-1}$ due to $\mathrm{NH}_{2}$ and two CN functions, respectively. ( Scheme 4).

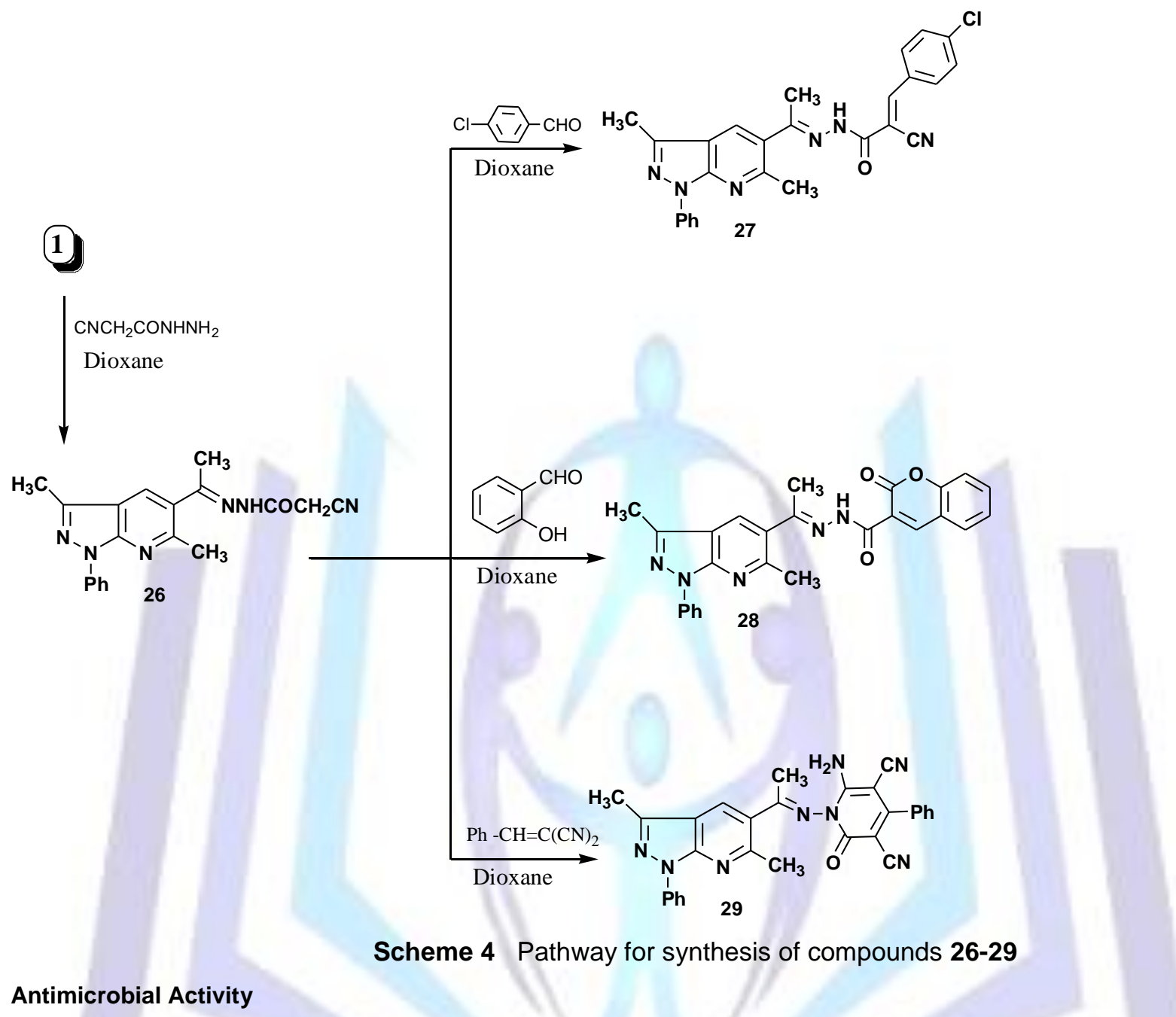

The antimicrobial activity of $\mathbf{2 2}$ selected compounds was screened against bacterial strains Staphylococcus aureus (AUMC B.54), Bacillus cereus (AUMC B.52) as gram-positive bacteria and Escherichia coli (AUMC B.53), Pseudomonas aeruginosa (AUMC B.73) as gram-negative bacteria and fungal strains Candida albicans (AUMC No.214), Aspergillus flavus (AUMC No.1276) using the agar well-diffusion method. ${ }^{31}$ The screening tests were carried out in triplicate and the results were expressed as a mean of three determinations. Chloramphenicol and Clotrimazole were used as standards. Data are represented as \% inhibition with reference to standards in (Table1). The results obtained from table 1 revealed that a comparison of the antimicrobial activity of the chalcone derivatives (2a-d), indicated that the para-chloro derivative 2c induced more activity against all of the tested microorganisms (76 - >100\% growth inhibition). The 5-isoxazolinyl derivative $\mathbf{4}$ was more potent than the 5-pyrazolinyl derivative 3 a and showed a good antifungal activity only against $C$. albicans (89\% growth inhibition) and $A$. flavus (79\% growth inhibition). The 5- pyrimidinylthione compound $5 \mathbf{b}\left(\mathrm{R}=\mathrm{CH}_{2} \mathrm{Ph}\right)$ showed a remarkable antimicrobial activity towards all of gram-positive bacteria ( $>100 \%$ growth inhibition against S.aureus) and also exhibited an excellent antifungal activity against C. albicans ( $100 \%$ growth inhibition). Formation of Schiff base of 5-aminopyrimidinyl derivative with benzaldehyde compound 8 showed only $>100 \%$ growth inhibition against C. albicans which was comparable to clotrimazole. The $\mathrm{N}$-thiazolylpyrazolinyl derivative $10 \mathrm{a}\left(\mathrm{R}=\mathrm{CH}_{3}\right)$ showed a remarkable antibacterial activity against gram-negative bacteria species E.coli ( $>100 \%$ growth inhibition) and $P$. aeruoginosa (83\% growth inhibition) and also exhibited a strong antifungal activity against C. albicans (>100\% growth inhibition). While, its phenyl isomer $\mathbf{1 0 b}(\mathrm{R}=\mathrm{Ph})$ was inactive towards most of the tested microorganisms. Similarly, the $\mathrm{N}$ phenyl thiazolylhydrazine derivative $\mathbf{1 2 b}(\mathrm{R}=\mathrm{Ph})$ did not show any activity against all of tested bacteria and fungi species. Whereas, it's $\mathrm{N}$-methyl derivative 12a $\left(\mathrm{R}=\mathrm{CH}_{3}\right)$ showed an excellent antimicrobial activity against all of tested bacteria and fungi species except $P$. aeruoginosa (95\% growth inhibition against E.coli). Building up a new 1,2,4-triazolidine thione compound 13 showed $>100 \%$ growth inhibition activity against all of tested bacteria and fungi species except $P$. aeruoginosa. The S-alkylating product compound 14 exihibited only $86 \%$ growth inhibition against E.coli. The creation of a novel thiazolotriazole ring compound 16, enhanced the antimicrobial activity. 
Table 1: Antimicrobial activity of some pyrazolo[3,4-b]pyridine derivatives

\begin{tabular}{|c|c|c|c|c|c|c|}
\hline \multirow[t]{3}{*}{ Compounds } & \multicolumn{6}{|c|}{ Diameter of zone of inhibition $(\mathrm{mm}) / \%$ inhibition with reference to standard } \\
\hline & \multicolumn{2}{|c|}{ Gram-positive bacteria } & \multicolumn{2}{|c|}{ Gram- nagative bacteria } & \multicolumn{2}{|r|}{ Fungi } \\
\hline & S.aureus & B.cereus & E.coli & P.aeruoginosá & C.albicans & A. flavus \\
\hline $2 \mathbf{a}$ & - & $10(40)$ & $9(43)$ & - & $8(30)$ & - \\
\hline $\mathbf{2 b}$ & - & $8(32)$ & - & - & - & - \\
\hline $2 c$ & $17(85)$ & $19(76)$ & - & $16(89)$ & $34(>100)$ & $35(92)$ \\
\hline $2 d$ & - & - & - & - & $6(22)$ & - \\
\hline $3 a$ & - & - & - & - & $7(26)$ & - \\
\hline 4 & - & - & - & - & $24(89)$ & $30(79)$ \\
\hline $5 a$ & - & - & - & - & 9 (33) & - \\
\hline $5 b$ & $26(>100)$ & $21(84)$ & - & - & $31(>100)$ & - \\
\hline 8 & - & - & $11(52)$ & - & $29(>100)$ & $23(61)$ \\
\hline $10 a$ & - & - & $25(>100)$ & $15(83)$ & $30(>100)$ & $26(68)$ \\
\hline $10 b$ & - & - & - & - & - & $12(32)$ \\
\hline $12 a$ & $18(90)$ & $20(80)$ & 20(95 ) & - & $23(85)$ & $30(79)$ \\
\hline $12 b$ & - & - & - & - & - & - \\
\hline 13 & $25(>100)$ & $24(96)$ & $28(>100)$ & - & $32(>100)$ & $41(>100)$ \\
\hline 14 & - & - & $18(86)$ & - & - & - \\
\hline 16 & $14(70)$ & $22(88)$ & - & - & $31(>100)$ & $36(95)$ \\
\hline 18 & $22(>100)$ & $19(76)$ & $24(>100)$ & - & - & $32(84)$ \\
\hline 19 & - & - & - & - & $29(>100)$ & $40(>100)$ \\
\hline 22 & $8(40)$ & - & - & - & 11(41) & $10(26)$ \\
\hline 24 & - & - & - & - & - & - \\
\hline 25 & - & - & $28(>100)$ & $16(89)$ & $30(>100)$ & - \\
\hline 28 & & - & - & - & - & - \\
\hline Chloramphenicol & $20(100)$ & $25(100)$ & $21(100)$ & $18(100)$ & - & - \\
\hline Clotrimazole & - & $\ldots$ & & & $27(100)$ & $38(100)$ \\
\hline
\end{tabular}

*The percentage zone of inhibition was calculated against the bacterial and fungal strains. Solvent and negative control: DMSO (dimethylsulfoxide)- No inhibition observed

Thus, compound 16 showed a significant antibacterial activity against gram-positive only (88\% growth inhibition against B.cereus) and also exhibited an excellent antifungal activity ( $>100 \%$ growth inhibition against $C$. albicans). On the other hand, the comparison of the percentage of growth inhibition of the thiadiazole and selenadiazole 18 and 19 respectively, demonstrated that thiadiazole derivative $\mathbf{1 8}$ is more potent against all of tested bacteria and fungi species except $P$. aeruoginosa and C. albicans (>100\% growth inhibition against S.aureus and E.coli), while, the selenadiazole 19 showed only an excellent antifungal activity ( $>100 \%$ growth inhibition against $C$. albicans and $A$. flavus). Among all of the spiroindolyl derivatives 22, 24 and 25 only, the spiroindolylpyrimidinethione 25 showed a significant antibacterial activity against gram-negative species (>100\% growth inhibition against E.coli) and also exhibited $>100 \%$ growth inhibition against C. albicans. With respect to the formation of coumarin compound $\mathbf{2 8}$, it was devoid of growth inhibition against all of the tested microorganisms.

\section{CONCLUSION}

The present study research study reports the convienent synthesis, reactions and antimicrobial activity of a new series of pyrazolo[3,4-b]pyridines carrying biologically active heterocyclic entities. Their Screening results revealed that most of the tested compounds showed a remarkable to an excellent activities and might be helpful in the future development of pyrazolopyridines as analogues as a novel antimicrobial agents. 


\section{EXPERIMENTAL}

Melting points are uncorrected and determined using a Gallenkamp melting point apparatus. IR spectra were recorded on a Pye-Unicam SP 3-100 spectrophotometer using the $\mathrm{KBr}$ wafer technique. ${ }^{1} \mathrm{H}$ NMR spectra were recorded on a Varian EM-390 $90 \mathrm{MHz}$ spectrometer and on GNM-LA $(400 \mathrm{MHz})$ in DMSO-d $\mathrm{d}_{6}$ as solvent and TMS as internal standard. Chemical shifts are expressed in ppm. ${ }^{13} \mathrm{C}$ NMR spectra were measured on a Varian EM-200, $100 \mathrm{MHZ}$ spectrometer. Mass spectra were determined on a JEOL JMS-600 spectrometer. Elemental analyses were carried out at the Microanalytical Unit at the at Assiut University (Egypt). Compounds 1 was prepared according to literature procedure. ${ }^{26}$ Compounds $2 \mathrm{a}, 11$ and 17 were prepared were prepared as previously reported. ${ }^{27}$

General procedure for the synthesis of 1-(3,6-dimethyl-1-phenyl-1H-pyrazolo[3,4-b]pyridin-5-yl)-3substitutedphenylprop-2-en-1-one (2a-d).

A mixture of $1(0.002 \mathrm{~mol})$, appropriate aromatic aldehydes $(0.002 \mathrm{~mol})$ and potassium hydroxide solution $(5 \mathrm{~mL}, 25 \%)$ in ethanol $(25 \mathrm{~mL})$ was stirred overnight. The product was filtered off, washed with water, dried and crystallized from ethanol.

\section{1-(3,6-Dimethyl-1-phenyl-1H-pyrazolo[3,4-b]pyridin-5-yl)-3-(4-methoxyphenyl)prop-2-en-1-one (2b)}

A yellow crystals, 56\%, mp 134-136ㄷ. FT-IR (KBr) $\mathrm{v}_{\max } / \mathrm{cm}^{-1}$ : $3062(\mathrm{CH}$ - aromatic), 2985, 2930 (CH- aliphatic), 1682 $(\mathrm{C}=\mathrm{O}), 1585(\mathrm{C}=\mathrm{N}), 1439(\mathrm{C}=\mathrm{C}), 1070(\mathrm{C}-\mathrm{O}) ;{ }^{1} \mathrm{H}$ NMR $\delta 2.40\left(\mathrm{~s}, 3 \mathrm{H}, \mathrm{CH}_{3}\right), 2.55\left(\mathrm{~s}, 3 \mathrm{H}, \mathrm{CH}_{3}\right), 3.85(\mathrm{~s}, 3 \mathrm{H}, \mathrm{OCH}), 6.75(\mathrm{~s}$, $1 \mathrm{H}, \quad \mathrm{CH}$-ethylenic), $7.50-8.35(\mathrm{~m}, 12 \mathrm{H}, 9 \mathrm{H}$-aromatic $+1 \mathrm{H}, \mathrm{CH}$-ethylenic $+1 \mathrm{H}, \mathrm{CH}$-pyridine $)$, Anal. Calcd for $\mathrm{C}_{24} \mathrm{H}_{21} \mathrm{~N}_{3} \mathrm{O}_{2}$ (383.44): C, 75.18; $\mathrm{H}, 5.52 ; \mathrm{N}, 10.96$. Found: $\mathrm{C}, 75.51 ; \mathrm{H}, 5.88 ; \mathrm{N}, 11.25 \%$.

\section{1-(3,6-Dimethyl-1-phenyl-1H-pyrazolo[3,4-b]pyridin-5-yl)-3-(4-chlorophenyl)prop-2-en-1-one (2c)}

A yellow crystals, $83 \%$, mp 188-190ㄷ. FT-IR (KBr) $\mathrm{V}_{\max } / \mathrm{cm}^{-1}: 3080(\mathrm{CH}$ - aromatic), 2980, 2866(CH- aliphatic), 1690 $(\mathrm{C}=\mathrm{O}), 1570(\mathrm{C}=\mathrm{N}), 1490(\mathrm{C}=\mathrm{C}) ;{ }^{1} \mathrm{H}$ NMR $\delta 2.45\left(\mathrm{~s}, 3 \mathrm{H}, \mathrm{CH}_{3}\right), 2.68\left(\mathrm{~s}, 3 \mathrm{H}, \mathrm{CH}_{3}\right), 6.63(\mathrm{~s}, 1 \mathrm{H}, \mathrm{CH}$-ethylenic), 7.50-8.30 (m, $12 \mathrm{H}, 10 \mathrm{H}$-aromatic $+1 \mathrm{H}$, CH-ethylenic $+1 \mathrm{H}, \mathrm{CH}$-pyridine), Anal. Calcd. for $\mathrm{C}_{23} \mathrm{H}_{18} \mathrm{CIN}_{3} \mathrm{O}(387.86): \mathrm{C}, 71.22 ; \mathrm{H}, 4.68 ; \mathrm{Cl}$, 9.14; N, 10.83. Found: C, 71.48; H, 5.02; Cl, 9.37; N, 11.11\%.

\section{1-(3,6-Dimethyl-1-phenyl-1H-pyrazolo[3,4-b]pyridin-5-yl)-3-(4-nitrophenyl)prop-2-en-1-one (2d)}

A yellow crystals, 69\%, mp 208-210ㄷ. FT-IR (KBr) $\mathrm{v}_{\max } / \mathrm{cm}^{-1}: 3097(\mathrm{CH}$ - aromatic), 2953, 2880 (CH- aliphatic), 1678 $(\mathrm{C}=\mathrm{O}), 1565(\mathrm{C}=\mathrm{N}), 1469(\mathrm{C}=\mathrm{C}) ;{ }^{1} \mathrm{H}$ NMR $\delta 2.40$ (s, 3H, $\left.\mathrm{CH}_{3}\right), 2.70$ (s, 3H, $\left.\mathrm{CH}_{3}\right), 6.48$ (s, 1H, CH-ethylenic), $7.40-8.55$ (m, $12 \mathrm{H}, 10 \mathrm{H}$-aromatic $+1 \mathrm{H}$, CH-ethylenic $+1 \mathrm{H}, \mathrm{CH}$-pyridine), Anal. Calcd. for $\mathrm{C}_{23} \mathrm{H}_{18} \mathrm{~N}_{4} \mathrm{O}_{3}(398.41): \mathrm{C}, 69.34 ; \mathrm{H}, 4.55 ; \mathrm{N}$, 14.06. Found: C, $69.59 ; \mathrm{H}, 4.84 ; \mathrm{N}, 14.30 \%$.

General procedure for the synthesis of 5-[5-(4-chloro phenyl)-3,6-dimethyl--4,5-dihydro-1H (phenyl)-pyrazol-3-yl]1-phenyl-1H-pyrazolo[3,4-b]pyridine $(3 a, b)$

A mixture of chalcone $2 \mathrm{c}(0.001 \mathrm{~mol})$, hydrazine hydrate or phenyl hydrazine $(0.001 \mathrm{~mol})$ was heated under reflux for $4 \mathrm{~h}$, in $20 \mathrm{~mL}$ ethanol then cooled and the residual material was filtered off and recrystallized from ethanol.

\section{5-[5-(4-chlorophenyl)-3,6-dimethyl--4,5-dihydro-1H-pyrazol-3-yl]-1-phenyl-1H-pyrazolo[3,4-b]pyridine (3a)}

A yellow crystals, 66\%, mp 159-161ำ. FT-IR (KBr) $\mathrm{v}_{\max } / \mathrm{cm}^{-1}: 3100(\mathrm{NH}), 3020$ (CH- aromatic), 2980 (CH- aliphatic), $1530(\mathrm{C}=\mathrm{N}), 1440(\mathrm{C}=\mathrm{C})$; ${ }^{1} \mathrm{H}$ NMR $\delta 2.48\left(\mathrm{~s}, 3 \mathrm{H}, \mathrm{CH}_{3}\right), 2.66\left(\mathrm{~s}, 3 \mathrm{H}, \mathrm{CH}_{3}\right), 3.12\left(\mathrm{dd}, J_{1}=4.75 \mathrm{~Hz}, J_{2}=7.70,1 \mathrm{H}, \mathrm{CH}_{2}\right.$ pyrazoline), $4.21\left(\mathrm{dd}, J_{1}=3.80 \mathrm{~Hz}, J_{2}=8.20,1 \mathrm{H}, \mathrm{CH}_{2}\right.$-pyrazoline), 4.85 (dd, $J_{1}=1.60 \mathrm{~Hz}, J_{2}=8.90,1 \mathrm{H}, \mathrm{CH}$-pyrazoline) 7.70 $8.45(\mathrm{~m}, 11 \mathrm{H}, 10 \mathrm{H}$-aromatic $+1 \mathrm{H}, \mathrm{CH}$-pyridine $), 8.80(\mathrm{~s}, 1 \mathrm{H}, \mathrm{NH}) ; \mathrm{MS} \mathrm{m} / \mathrm{z}(\%): 401.46\left(\mathrm{M}^{+}\right.$, 100); Anal. Calcd. for $\mathrm{C}_{23} \mathrm{H}_{20} \mathrm{CIN}_{5}$ (401.89): C, 68.74; H, 5.02; Cl, 8.82; N, 17.43. Found: C, 69.03; H, 5.38; Cl, 9.13; N, 17.70\%.

\section{5-[5-(4-Chlorophenyl)-3,6-dimethyl--4,5-dihydro-1-phenylpyrazol-3-yl]-1-phenyl-1H-pyrazolo[3,4-b]pyridine (3b)}

A yellow crystals, 71\%, mp 223-225으. FT-IR (KBr) $\mathrm{v}_{\max } / \mathrm{cm}^{-1}: 3066$ ( $\mathrm{CH}$ - aromatic), $2969(\mathrm{CH}-$ aloiphatic), $1546(\mathrm{C}=\mathrm{N})$, $1481(\mathrm{C}=\mathrm{C}) ;{ }^{1} \mathrm{H}$ NMR $\delta 2.55\left(\mathrm{~s}, 3 \mathrm{H}, \mathrm{CH}_{3}\right), 2.69\left(\mathrm{~s}, 3 \mathrm{H}, \mathrm{CH}_{3}\right), 3.25$ (dd, $\left.J_{1}=5.30 \mathrm{~Hz}, \mathrm{~J}_{2}=2.70,1 \mathrm{H}, \mathrm{CH}_{2}-\mathrm{pyrazoline}\right), 4.15$ (dd, $J_{1}=3.60 \mathrm{~Hz}, J_{2}=1.80,1 \mathrm{H}, \mathrm{CH}_{2}$-pyrazoline), $5.27\left(\mathrm{dd}, J_{1}=8.40 \mathrm{~Hz}, J_{2}=1.20,1 \mathrm{H}, \mathrm{CH}\right.$-pyrazoline) $7.65-8.55(\mathrm{~m}, 15 \mathrm{H}, 14 \mathrm{H}-$ aromatic $+1 \mathrm{H}$, CH-pyridine); Anal. Calcd. for $\mathrm{C}_{29} \mathrm{H}_{24} \mathrm{ClN}_{5}$ (477.99): C, 72.87; H, 5.06; Cl, 7.42; N, 14.65. Found: C, 73.12; $\mathrm{H}, 5.33 ; \mathrm{Cl}, 7.65 ; \mathrm{N}, 14.84 \%$.

\section{5-[5-(4-chlorophenyl)-4,5-dihydro-1,2-isoxazol-3-yl]-3,6-dimethyl-1-phenyl-1H-pyrazolo[3,4-b]pyridine (4)}

A mixture of chalcone $2 \mathrm{c}(0.38 \mathrm{~g}, 0.001 \mathrm{~mol})$, hydroxylamine hydrochloride $(0.07 \mathrm{~g}, 0.001 \mathrm{~mol})$ and anhydrous sodium acetate $(1 \mathrm{~g})$ was refluxed in ethanol $(20 \mathrm{~mL})$ for $6 \mathrm{~h}$, then allowed to cool and poured into cold water. The precipitated product thus formed was collected by filtration, dried and recrystallized from methanol to give compound 4 as yellow 


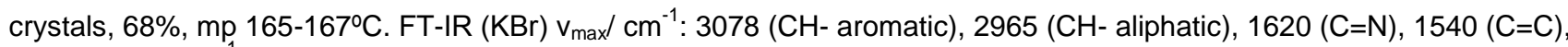
1509 (=C=N-O); ${ }^{1} \mathrm{H}$ NMR $\delta 2.37\left(\mathrm{~s}, 3 \mathrm{H}, \mathrm{CH}_{3}\right), 2.64\left(\mathrm{~s}, 3 \mathrm{H}, \mathrm{CH}_{3}\right), 3.15\left(\mathrm{dd}, J_{1}=5.30 \mathrm{~Hz}, \mathrm{~J}_{2}=2.70,1 \mathrm{H}, \mathrm{CH}_{2}\right.$ - isoxazol), 3.78 $\left(\mathrm{dd}, J_{1}=3.60 \mathrm{~Hz}, J_{2}=1.80,1 \mathrm{H}, \mathrm{CH}_{2}\right.$ - isoxazol), $5.15\left(\mathrm{dd}, J_{1}=8.40 \mathrm{~Hz}, J_{2}=1.20,1 \mathrm{H}, \mathrm{CH}\right.$ - isoxazol) $7.65-8.55(\mathrm{~m}, 15 \mathrm{H}, 14 \mathrm{H}-$ aromatic $+1 \mathrm{H}, \mathrm{CH}$-pyridine); Anal. Calcd. for $\mathrm{C}_{29} \mathrm{H}_{24} \mathrm{ClN}_{5}$ (477.99): C, 72.87; H, 5.06; Cl, 7.42; N, 14.65. Found: C, 73.12; $\mathrm{H}, 5.33$; $\mathrm{Cl}, 7.65$; N, 14.84\%.

General procedure for the synthesis of 4-(4-chlorophenyl)-6-(3,6-dimethyl-1-phenyl-1H-pyrazolo[3,4-b]pyridin-5yl)pyrimidin-2-ol (thiol) $(5 a, b)$

A mixture of chalcone $2 \mathrm{c}(0.02 \mathrm{~mol})$, urea or thiourea $(0.02 \mathrm{~mol})$ were dissolved in ethanolic sodium hydroxide $(5 \%, 10$ $\mathrm{mL}$ ) was stirred about 2-3 hours with a magnetic stirrer. This was then poured into $400 \mathrm{ml}$ of cold water with continuous stirring for an hour and then kept in refrigerator for 24 hours. The precipitate obtained was filtered, washed and recrystallized ethanol.

\section{4-(4-chlorophenyl)-6-(3,6-dimethyl-1-phenyl-1H-pyrazolo[3,4-b]pyridin-5-yl)pyrimidin-2-ol (5a)}

A pale brown crystals, 71\%, mp 236-238으. FT-IR (KBr) $\mathrm{v}_{\max } / \mathrm{cm}^{-1}: 3460(\mathrm{OH}), 3060(\mathrm{CH}$ - aromatic), $2982(\mathrm{CH}-$ aliphatic), $1606(\mathrm{C}=\mathrm{N}), 1472(\mathrm{C}=\mathrm{C}) ;{ }^{1} \mathrm{H}$ NMR $\delta 2.61\left(\mathrm{~s}, 3 \mathrm{H}, \mathrm{CH}_{3}\right), 2.74\left(\mathrm{~s}, 3 \mathrm{H}, \mathrm{CH}_{3}\right), 3.20(\mathrm{~s}, 1 \mathrm{H}, \mathrm{OH}), 7.25(\mathrm{~s}, 1 \mathrm{H}, \mathrm{CH}$-pyrimidine), 7.55-8.60 (m, $10 \mathrm{H}, 9 \mathrm{H}$-aromatic $+1 \mathrm{H}, \mathrm{CH}$-pyridine); Anal. Calcd. for $\mathrm{C}_{24} \mathrm{H}_{18} \mathrm{ClN}_{5} \mathrm{O}$ (427.89): $\mathrm{C}, 67.37 ; \mathrm{H}, 4.24 ; \mathrm{Cl}, 8.29 ; \mathrm{N}$, 16.37. Found: C, $67.71 ; \mathrm{H}, 4.48 ; \mathrm{Cl}, 8.52 ; \mathrm{N}, 16.39 \%$.

4-(4-chlorophenyl)-6-(3,6-dimethyl-1-phenyl-1H-pyrazolo[3,4-b]pyridin-5-yl)pyrimidin-2-thiol (5b)

A pale brown crystals, 79\%, mp 293-295ㄷ. FT-IR (KBr) $\mathrm{v}_{\max } / \mathrm{cm}^{-1}: 3372,3267(\mathrm{NH}), 3061(\mathrm{CH}-$ aromatic), $2995(\mathrm{CH}-$ aliphatic), $1626(\mathrm{C}=\mathrm{N}), 1375(\mathrm{C}=\mathrm{S}) ;{ }^{1} \mathrm{H}$ NMR $\delta 2.55\left(\mathrm{~s}, 3 \mathrm{H}, \mathrm{CH}_{3}\right), 2.79\left(\mathrm{~s}, 3 \mathrm{H}, \mathrm{CH}_{3}\right), 3.40(\mathrm{~s}, 1 \mathrm{H}, \mathrm{SH}), 7.50-8.54(\mathrm{~m}, 11 \mathrm{H}$, $9 \mathrm{H}$-aromatic $+1 \mathrm{H}, \mathrm{CH}$-pyridine $+1 \mathrm{H}, \mathrm{CH}$ - pyrimidine); Anal. Calcd. for $\mathrm{C}_{24} \mathrm{H}_{18} \mathrm{CIN}_{5} \mathrm{~S}$ (443.95): C, 64.93; $\mathrm{H}, 4.09 ; \mathrm{Cl}, 7.99$; N, 15.78; S, 7.22\%. Found: C, 65.28; H, 4.24; Cl, 8.30; N, 16.07; S, 7.53\%.

General procedure for the synthesis of 2-substituted thio-4-(4-chlorophenyl)-6-(3,6-dimethyl-1-phenyl-1Hpyrazolo[3,4-b]pyridin-5-yl)pyrimidine $(6 a, b)$

A mixture of $5 \mathrm{~b}(0.01 \mathrm{~mol})$, ethyl iodide or benzyl bromide $(0.01 \mathrm{~mol})$ in ethanol $(40 \mathrm{~mL})$ was refluxed in the presence of anhydrous sodium acetate $(0.9 \mathrm{~g}, 0.011 \mathrm{~mol})$ for $4 \mathrm{~h}$. The solid product separated from the hot mixture was filtered off, washed with water and recrystallized from ethanol

4-(4-Chlorophenyl)-2-ethylthio-4-(4-chlorophenyl)-6-(3,6-dimethyl-1-phenyl-1H-pyrazolo[3,4-b]pyridin-5-yl)pyrimidine (6a)

A pale yellow crystal, 68\%, mp 167-169ㅇ․ FT-IR (KBr) $\mathrm{v}_{\max } / \mathrm{cm}^{-1}: 3088(\mathrm{CH}$ - aromatic), $2975(\mathrm{CH}$-aliphatic), $1620(\mathrm{C}=\mathrm{N})$, 1498 (C=C), 1266 (C-S); ${ }^{1} \mathrm{H}$ NMR $\delta 1.25$ (t, 3H, SCH$\left.-\underline{C H}_{3}\right), 2.45\left(\mathrm{~s}, 3 \mathrm{H}, \mathrm{CH}_{3}\right), 2.60\left(\mathrm{~s}, 3 \mathrm{H}, \mathrm{CH}_{3}\right), 4.00\left(\mathrm{q}, 2 \mathrm{H}, \mathrm{SCH}_{2} \mathrm{CH}_{3}\right)$, $7.40\left(\mathrm{~s}, 1 \mathrm{H}, \mathrm{CH}\right.$-pyrimidine), $7.60-8.75\left(\mathrm{~m}, 10 \mathrm{H}, 9 \mathrm{H}\right.$-aromatic $+1 \mathrm{H}, \mathrm{CH}$-pyridine); Anal. Calcd. for $\mathrm{C}_{26} \mathrm{H}_{22} \mathrm{CIN}_{5} \mathrm{~S}(472.13)$ : C, 66.16; H, 4.70; Cl, 5.71; N, 14.84; S, 6.79. Found: C, 66.48; H, 5.06; Cl, 5.82; N, 15.13; S, 7.12\%.

\section{2-Benzylthio-4-(4-chlorophenyl)-6-(3,6-dimethyl-1-phenyl-1H-pyrazolo[3,4-b]pyridin-5-yl)pyrimidine (6b)}

A pale yellow crystals, 61\%, mp 157-1590. FT-IR (KBr) $\mathrm{V}_{\max } / \mathrm{cm}^{-1}: 3090(\mathrm{CH}-$ aromatic), 2874 (CH- aliphatic), 1615 $(\mathrm{C}=\mathrm{N}), 1398(\mathrm{C}=\mathrm{C}), 1244(\mathrm{C}-\mathrm{S}) ;{ }^{1} \mathrm{H}$ NMR $\delta 2.40\left(\mathrm{~s}, 3 \mathrm{H}, \mathrm{CH}_{3}\right), 2.65$ (s, 3H, $\left.\mathrm{CH}_{3}\right), 4.15(\mathrm{~s}, 2 \mathrm{H}, \mathrm{S} \underline{\mathrm{CH}} 2 \mathrm{Ph}), 7.35(\mathrm{~s}, 1 \mathrm{H}, \mathrm{CH}-$ pyrimidine), $7.55-8.60\left(\mathrm{~m}, 16 \mathrm{H}, 15 \mathrm{H}\right.$-aromatic $+1 \mathrm{H}, \mathrm{CH}$-pyridine); Anal. Calcd. for $\mathrm{C}_{31} \mathrm{H}_{24} \mathrm{CIN}_{5} \mathrm{~S}(534.07): \mathrm{C}, 69.72 ; \mathrm{H}$, 4.53; Cl, 6.64; N, 13.11; S, 6.00. Found: C, 66.93; H, 5.00; Cl, 6.77; N, 13.39; S, 6.21\%.

\section{2-Amino-4-(4-chlorophenyl)-6-(3,6-dimethyl-1-phenyl-1H-pyrazolo[3,4-b]pyridin-5-yl)pyrimidine (7)}

A mixture of chalcone $2 \mathrm{c}(0.76 \mathrm{~g}, 0.002 \mathrm{~mol})$, guanidine hydrochloride $(0.19 \mathrm{~g}, 0.002 \mathrm{~mol})$ and ethanolic sodium hydroxide $(10 \%, 10 \mathrm{~mL})$ was refluxed for $2-3 \mathrm{~h}$. After cooling, the solid formed was filtered off, air dried and recrystallized from absolute ethanol to give compound 7 as brown crystals, $71 \%, \mathrm{mp} 305-307^{\circ} \mathrm{C}$. FT-IR $(\mathrm{KBr}) \mathrm{v}_{\max } / \mathrm{cm}^{-1}: 3320,3290\left(\mathrm{NH}_{2}\right)$, 3035 (CH-aromatic), 2880 (CH- aliphatic), $1627(\mathrm{C}=\mathrm{N}), 1526(\mathrm{C}=\mathrm{C}) ;{ }^{1} \mathrm{H}$ NMR $\delta 2.54\left(\mathrm{~s}, 3 \mathrm{H}, \mathrm{CH}_{3}\right), 2.63\left(\mathrm{~s}, 3 \mathrm{H}, \mathrm{CH}_{3}\right), 6.95$ (s, $1 \mathrm{H}, \mathrm{CH}$-pyrimidine), 7.55-8.45 (m, 10H, 9H-aromatic $+1 \mathrm{H}, \mathrm{CH}$-pyridine), $9.45\left(\mathrm{~s}, 2 \mathrm{H}, \mathrm{NH}_{2}, \mathrm{D}_{2} \mathrm{O}\right.$ exchangeable); Anal. Calcd. for $\mathrm{C}_{24} \mathrm{H}_{19} \mathrm{CIN}_{6}$ (426.9): C, 67.52; H, 4.49; Cl, 8.30; N, 19.69. Found: C, 67.83; H, 4.79; Cl, 8.60; N, 19.77\%.

\section{2-(4-Chlorobenzylideneamino)-4-(4-chlorophenyl)-6-(3,6-dimethyl-1-phenyl-1H-pyrazolo[3,4-b]pyridin-5- yl)pyrimidine (8)}

A mixture of compound $7(0.43 \mathrm{~g}, 0.001 \mathrm{~mol})$ and benzaldehyde $(0.11 \mathrm{~g}, 0.001 \mathrm{~mol})$ was stirred under reflux in methanol $(30 \mathrm{ml})$ in the presence of a few drops of glacial acetic acid for $5 \mathrm{~h}$. The reaction mixture was allowed to cool to room temperature, poured into water, whereby a solid formed that was filtered off and crystallized from dioxane to give 
compound 8 as brown crystals, 68\%, mp 263-265ㅇ․ FT-IR (KBr) $\mathrm{V}_{\max } / \mathrm{cm}^{-1}: 3018(\mathrm{CH}-$ aromatic), 2890 (CH- aliphatic), $1633(\mathrm{C}=\mathrm{N}), 1525(\mathrm{C}=\mathrm{C}) ;{ }^{1} \mathrm{H}$ NMR $\delta 2.48\left(\mathrm{~s}, 3 \mathrm{H}, \mathrm{CH}_{3}\right), 2.70\left(\mathrm{~s}, 3 \mathrm{H}, \mathrm{CH}_{3}\right), 7.35-8.35(\mathrm{~m}, 17 \mathrm{H}, 14 \mathrm{H}$-aromatic $+1 \mathrm{H}, \mathrm{CH}-$ pyridine $+1 \mathrm{H}, \mathrm{CH}$-pyrimidine $+1 \mathrm{H}, \mathrm{CH}=\mathrm{N})$; Anal. Calcd. for $\mathrm{C}_{31} \mathrm{H}_{23} \mathrm{CIN}_{6}(515.01)$ : $\mathrm{C}, 72.30 ; \mathrm{H}, 4.50 ; \mathrm{Cl}, 6.88 ; \mathrm{N}, 16.32$. Found: C, 72.58; H, 4.90; Cl, 7.10; N, 16.62\%.

\section{5-(4-Chlorophenyl)-4,5-dihydro-3-(3,6-dimethyl-1-phenyl-1H-pyrazolo[3,4-b]pyridin-5-yl)pyrazole-1- carbothioamide (9)}

A mixture chalcone $2 \mathrm{c}(0.76 \mathrm{~g}, 0.002 \mathrm{~mol})$ and thiosemicarbazide $(0.18 \mathrm{~g}, 0.001 \mathrm{~mol})$ in and ethanolic sodium hydroxide $(25 \%, 10 \mathrm{~mL})$ was refluxed for $3 \mathrm{~h}$. The reaction mixture was allowed to cool to room temperature, whereby a solid formed that was filtered off and crystallized from DMF $-\mathrm{H}_{2} \mathrm{O}$ to give compound 9 as yellow crystals, 70\%, mp 202-204으. $\mathrm{CT}$-IR $(\mathrm{KBr}) \mathrm{V}_{\max } / \mathrm{cm}^{-1}: 3425,3250\left(\mathrm{NH}_{2}\right), 3057(\mathrm{CH}-$ aromatic), $2927(\mathrm{CH}-$ aliphatic), $1580(\mathrm{C}=\mathrm{N}), 1460(\mathrm{C}=\mathrm{C}), 1370(\mathrm{C}=\mathrm{S})$, $1120(\mathrm{C}-\mathrm{N}) ;{ }^{1} \mathrm{H}$ NMR $\delta 2.52\left(\mathrm{~s}, 3 \mathrm{H}, \mathrm{CH}_{3}\right), 2.70\left(\mathrm{~s}, 3 \mathrm{H}, \mathrm{CH}_{3}\right), 3.46$ (dd, $J_{1}=4.59 \mathrm{~Hz}, J_{2}=8.25,1 \mathrm{H}, \mathrm{CH}_{2}$-pyrazoline), $4.0(\mathrm{dd}$, $J_{1}=4.68 \mathrm{~Hz}, J_{2}=8.45,1 \mathrm{H}, \mathrm{CH}_{2}$-pyrazoline), 5.15 (dd, $J_{1}=1.68 \mathrm{~Hz}, J_{2}=8.85,1 \mathrm{H}, \mathrm{CH}$-pyrazoline), $6.70\left(\mathrm{~s}, 1 \mathrm{H}, \mathrm{NH}_{2}\right) ; 7.45-$ $8.30\left(\mathrm{~m}, 10 \mathrm{H}, 9 \mathrm{H}\right.$-aromatic $+1 \mathrm{H}, \mathrm{CH}$-pyridine); Anal. Calcd. for $\mathrm{C}_{24} \mathrm{H}_{21} \mathrm{ClN}_{6} \mathrm{~S}$ (460.98): $\mathrm{C}, 62.53 ; \mathrm{H}, 4.59 ; \mathrm{Cl}, 7.69 ; \mathrm{N}$, 18.23; S, 6.96. Found: C, 62.82; H, 5.01; Cl, 8.01; N, 18.47; S, 7.16\%; MS m/z (\%): $460.42\left(\mathrm{M}^{+}, 76\right)$.

\section{General procedure for the synthesis of 5-(4-Chlorophenyl)-4,5-dihydro-1-(4-substitutedthiazol-2-yl)-1-H-pyrazol-3- yl)-3,6-dimethyl-1-phenyl-1H-pyrazolo[3,4-b]pyridines $(10 a, b)$}

A mixture of $9(0.01 \mathrm{~mol})$, chloroacetone or phenacyl bromide $(0.01 \mathrm{~mol})$ in ethanol $(50 \mathrm{~mL})$ was refluxed in the presence of anhydrous sodium acetate $(0.9 \mathrm{~g}, 0.011 \mathrm{~mol})$ for $4 \mathrm{~h}$. The solid product separated from the hot mixture was filtered off, washed with water and recrystallized from the proper solvent.

5-(4-Chlorophenyl)-4,5-dihydro-1-(4-methylthiazol-2-yl)-1-H-pyrazol-3-yl)-3,6-dimethyl-1-phenyl-1H-pyrazolo[3,4-b]pyridine (10a).

A yellow crystals $(\mathrm{EtOH}), 70 \%, \mathrm{mp} 325-327^{\circ} \mathrm{C}$. FT-IR $(\mathrm{KBr}) \mathrm{v}_{\max } / \mathrm{cm}^{-1}: 3066(\mathrm{CH}-$ aromatic), 2947 (CH- aliphatic), 1626 $(\mathrm{C}=\mathrm{N}), 1562(\mathrm{C}=\mathrm{C}) ;^{1} \mathrm{H}$ NMR $\delta 2.55\left(\mathrm{~s}, 3 \mathrm{H}, \mathrm{CH}_{3}\right), 2.77\left(\mathrm{~s}, 3 \mathrm{H}, \mathrm{CH}_{3}\right), 3.19$ (dd, $J_{1}=5.22 \mathrm{~Hz}, J_{2}=7.35,1 \mathrm{H}, \mathrm{CH}_{2}-\mathrm{pyrazoline}$ ), $3.65\left(\mathrm{dd}, J_{1}=3.75 \mathrm{~Hz}, J_{2}=9.31,1 \mathrm{H}, \mathrm{CH}_{2}\right.$-pyrazoline), 4.75 (dd, $J_{1}=1.45 \mathrm{~Hz}, \mathrm{~J}_{2}=8.52,1 \mathrm{H}, \mathrm{CH}$-pyrazoline), $6.45(\mathrm{~s}, 1 \mathrm{H}, \mathrm{CH}-$ thiazole); $7.30-8.47\left(\mathrm{~m}, 10 \mathrm{H}, 9 \mathrm{H}\right.$-aromatic $+1 \mathrm{H}$, CH-pyridine). Anal. Calcd. for $\mathrm{C}_{27} \mathrm{H}_{23} \mathrm{CIN} \mathrm{N}_{6} \mathrm{~S}(499.03): \mathrm{C}, 64.98 ; \mathrm{H}, 4.65$; Cl, 7.10; N, 16.84; S, 6.43. Found: C, 65.12; H, 4.98; Cl, 7.38; N, 17.09; S, 6.49\%.

5-(4-Chlorophenyl)-4,5-dihydro-1-(4-phenylthiazol-2-yl)-1-H-pyrazol-3-yl)-3,6-dimethyl-1-phenyl-1H-pyrazolo[3,4b]pyridines (10b)

A yellow crystals (Dioxane),71\%, mp 217-219으. FT-IR (KBr) $\vee_{\max } / \mathrm{cm}^{-1}: 3051$ (CH- aromatic), 2962 (CH- aliphatic), 1637 $(\mathrm{C}=\mathrm{N}), 1581(\mathrm{C}=\mathrm{C}) ;{ }^{1} \mathrm{H}$ NMR $\delta 2.59\left(\mathrm{~s}, 3 \mathrm{H}, \mathrm{CH}_{3}\right), 2.70\left(\mathrm{~s}, 3 \mathrm{H}, \mathrm{CH}_{3}\right), 3.08\left(\mathrm{dd}, \mathrm{J}_{1}=5.17 \mathrm{~Hz}, \mathrm{~J}_{2}=8.12,1 \mathrm{H}, \mathrm{CH}_{2}-\mathrm{pyrazoline}\right)$, $3.81\left(\mathrm{dd}, J_{1}=3.90 \mathrm{~Hz}, J_{2}=9.65,1 \mathrm{H}, \mathrm{CH}_{2}\right.$-pyrazoline), 5.38 (dd, $J_{1}=0.87 \mathrm{~Hz}, \mathrm{~J}_{2}=6.88,1 \mathrm{H}, \mathrm{CH}$-pyrazoline), $6.20(\mathrm{~s}, 1 \mathrm{H}, \mathrm{CH}-$ thiazole); $7.10-8.55\left(\mathrm{~m}, 15 \mathrm{H}, 14 \mathrm{H}\right.$-aromatic $+1 \mathrm{H}$, CH-pyridine). Anal. Calcd. for $\mathrm{C}_{32} \mathrm{H}_{25} \mathrm{CIN}_{6} \mathrm{~S}(561.1): \mathrm{C}, 68.50 ; \mathrm{H}, 4.49$; Cl, 6.32; N, 14.98; S, 5.71. Found: C, 68.83; H, 4.67; Cl, 6.59; N, 15.27; S, 5.95\%.

General procedure for the synthesis of 1-(1-(3,6-dimethyl-1-phenyl-1H-pyrazolo[3,4-b]pyridine-5-yl)ethylidene)-2(4-substitutedthiazol-2-yl)hydrazine $(12 a, b)$

A mixture of thiosemicarbazone $11(0.001 \mathrm{~mol})$, chloroacetone or phenacyl bromide $(0.001 \mathrm{~mol})$ in ethanol $(30 \mathrm{~mL}) \mathrm{was}$ refluxed in the presence of anhydrous sodium acetate $(0.9 \mathrm{~g}, 0.011 \mathrm{~mol})$ for $4 \mathrm{~h}$. The solid product separated from the hot mixture was filtered off, washed with water and recrystallized from the proper solvent.

1-(1-(3,6-Dimethyl-1-phenyl-1H-pyrazolo[3,4-b]pyridine-5-yl)ethylidene)-2-(4-methylthiazol-2-yl)hydrazine (12a)

A yellow crystals $(\mathrm{MeOH}), 71 \%$, mp 292-294으. FT-IR $(\mathrm{KBr}) \mathrm{v}_{\max } / \mathrm{cm}^{-1}: 3290(\mathrm{NH}), 3060(\mathrm{CH}-$ aromatic), $2887(\mathrm{CH}-$ aliphatic), $1629(\mathrm{C}=\mathrm{N}), 1567(\mathrm{C}=\mathrm{C}) ;{ }^{1} \mathrm{H}$ NMR $\delta 1.25\left(\mathrm{~s}, 3 \mathrm{H}, \mathrm{CH}_{3}\right), 2.50\left(\mathrm{~s}, 3 \mathrm{H}, \mathrm{CH}_{3}\right), 2.65\left(\mathrm{~s}, 3 \mathrm{H}, \mathrm{CH}_{3}\right), 3.08\left(\mathrm{~s}, 3 \mathrm{H}, \mathrm{CH}_{3}\right)$, $6.25\left(\mathrm{~s}, 1 \mathrm{H}, \mathrm{CH}\right.$-thiazole); $6.95-8.45(\mathrm{~m}, 6 \mathrm{H}, 5 \mathrm{H}$-aromatic $+1 \mathrm{H}, \mathrm{CH}$-pyridine $), 9.35\left(\mathrm{~s}, 1 \mathrm{H}, \mathrm{NH}, \mathrm{D}_{2} \mathrm{O}\right.$-exchangeable). Anal. Calcd. for $\mathrm{C}_{20} \mathrm{H}_{20} \mathrm{~N}_{6} \mathrm{~S}$ (376.48): C, 63.81; H, 5.35; N, 22.32; S, 8.52. Found: C, 63.96; H, 5.68; N, 22.42; S, 8.83\%.

\section{1-(1-(3,6-Dimethyl-1-phenyl-1H-pyrazolo[3,4-b]pyridine-5-yl)ethylidene)-2-(4-phenylthiazol-2-yl)hydrazine (12b)}

A yellow crystals (Acetone $\left./ \mathrm{H}_{2} \mathrm{O}\right), 67 \%$, mp 225-227으. FT-IR $(\mathrm{KBr}) \mathrm{V}_{\max } / \mathrm{cm}^{-1}: 3325(\mathrm{NH}), 3048(\mathrm{CH}-$ aromatic), 2966 (CH- aliphatic), $1634(\mathrm{C}=\mathrm{N}), 1536(\mathrm{C}=\mathrm{C}) ;{ }^{1} \mathrm{H}$ NMR $\delta 0.95\left(\mathrm{~s}, 3 \mathrm{H}, \mathrm{CH}_{3}\right), 2.45\left(\mathrm{~s}, 3 \mathrm{H}, \mathrm{CH}_{3}\right), 2.75\left(\mathrm{~s}, 3 \mathrm{H}, \mathrm{CH}_{3}\right), 6.70(\mathrm{~s}, 1 \mathrm{H}$, $\mathrm{CH}$-thiazole); $7.37-8.35\left(\mathrm{~m}, 11 \mathrm{H}, 10 \mathrm{H}\right.$-aromatic $+1 \mathrm{H}, \mathrm{CH}$-pyridine), $9.15\left(\mathrm{~s}, 1 \mathrm{H}, \mathrm{NH}, \mathrm{D}_{2} \mathrm{O}\right.$-exchangeable). Anal. Calcd. for $\mathrm{C}_{25} \mathrm{H}_{22} \mathrm{~N}_{6} \mathrm{~S}$ (438.55): C, 68.47; H, 5.06; N, 19.16; S, 7.32. Found: C, 68.69; H, 5.41; N, 19.36; S, 7.57\%. 
5-Methyl-5-(3,6-dimethyl-1-phenyl-1H-pyrazolo[3,4-b]pyridine-5-yl)-1,2,4-triazolidine-3-thione (13)

\section{Method A}

A mixture of $11(0.34 \mathrm{~g}, 0.001 \mathrm{~mol})$ in methanol $(20 \mathrm{~mL})$ and hydrochloric acid $(1.5 \mathrm{~mL})$ was heated on the water-bath for 30 minutes. The solid obtained after cooling was filtered off, washed with water till acid-free then crystallized from methanol to give compound 13as yellow crystals, $62 \%$, mp 145-147으. FT-IR $(\mathrm{KBr}) \mathrm{v}_{\max } / \mathrm{cm}^{-1}: 3360,3345$ and $3220(\mathrm{NH})$, $3050\left(\mathrm{CH}\right.$ - aromatic stretch), 2980 (CH- aliphatic stretch), $2720(\mathrm{C}=\mathrm{S}), 1575(\mathrm{C}=\mathrm{N}) ;{ }^{1} \mathrm{H}$ NMR $\delta 1.55\left(\mathrm{~s}, 3 \mathrm{H}, \mathrm{CH}_{3}\right), 2.0(\mathrm{~s}$, $1 \mathrm{H}, \mathrm{NH}), 2.15(\mathrm{~s}, 1 \mathrm{H}, \mathrm{NH}), 2.25(\mathrm{~s}, 1 \mathrm{H}, \mathrm{NH}), 2.50\left(\mathrm{~s}, 3 \mathrm{H}, \mathrm{CH}_{3}\right), 2.65\left(\mathrm{~s}, 3 \mathrm{H}, \mathrm{CH}_{3}\right), 7.35-8.20(\mathrm{~m}, 6 \mathrm{H}, 5 \mathrm{H}$-aromatic $+1 \mathrm{H}$, $\mathrm{CH}$-pyridine), Anal. Calcd. for $\mathrm{C}_{17} \mathrm{H}_{18} \mathrm{~N}_{6} \mathrm{~S}$ (338.47): C, 60.33; $\mathrm{H}, 5.36 ; \mathrm{N}, 24.83 ; \mathrm{S}, 9.47$. Found: $\mathrm{C}, 60.71 ; \mathrm{H}, 5.59 ; \mathrm{N}, 25.12$; S, $9.68 \%$; MS: $m / z 338.19\left(\mathrm{M}^{+}, 68\right)$.

\section{Method B}

A mixture $1(0.26 \mathrm{~g}, 0.001 \mathrm{~mol})$ and thiosemicarbazide $(0.09 \mathrm{~g}, 0.001 \mathrm{~mole})$ in methanol $(20 \mathrm{~mL})$ and hydrochloric acid $(1.5$ $\mathrm{mL}$ ) was heated on the water-bath for $2 \mathrm{~h}$. The solid obtained after cooling was filtered off, washed with water till acid-free then crystallized from methanol to give compound 13. All of analytical and spectral data are completely identical as described in method $A$.

\section{General procedure for the synthesis of 14 and 15}

A mixture of $13(0.01 \mathrm{~mol})$, 1-bromo-2-methoxyethane $(0.01 \mathrm{~mol})$ and $\mathrm{K}_{2} \mathrm{CO}_{3}(1.50 \mathrm{~g})$ in $20 \mathrm{~mL}$ DMF was stirred at $75-$ $80^{\circ} \mathrm{C}$ overnight. Then, $30 \mathrm{~mL}$ water was added and the resulting mixture was extracted with $\mathrm{CHCl}_{3}$. The organic layer wasseparated, washed with water $(4 \times 30 \mathrm{~mL})$, and dried over $\mathrm{Na}_{2} \mathrm{SO}_{4}$. After solvent evaporation, the residue was purified by column chromatography on silica gel using $\mathrm{CHCl}_{3}-\mathrm{CH}_{3} \mathrm{COOCH}_{3}(70: 30)$ as an eluent to afford 14 and 15.

\section{5-[5-(2-Methoxy-ethylsulfanyl)-3-methyl-3,4-dihydro-2H-[1,2,4]triazol-3-yl)-3,6-dimethyl-1-phenyl-1H-pyrazolo[3,4-} b]pyridine (14)

A yellow crystals, $79 \%$, mp 176-178으. FT-IR ( KBr) $\mathrm{V}_{\max } / \mathrm{cm}^{-1}: 3405$ and $3325(\mathrm{NH}), 3048(\mathrm{CH}-$ aromatic), $2966(\mathrm{CH}-$ aliphatic), $1611(\mathrm{C}=\mathrm{N}) 1589(\mathrm{C}=\mathrm{C}), 1226(\mathrm{C}-\mathrm{O}) ;{ }^{1} \mathrm{H}$ NMR $\delta: 1.35\left(\mathrm{~s}, 3 \mathrm{H}, \mathrm{CH}_{3}\right), 2.2(\mathrm{~s}, 1 \mathrm{H}, \mathrm{NH}), 2.40\left(\mathrm{~s}, 3 \mathrm{H}, \mathrm{CH}_{3}\right), 2.65(\mathrm{~s}$, $\left.3 \mathrm{H}, \mathrm{CH}_{3}\right), 3.30\left(\mathrm{~s}, 3 \mathrm{H}, \mathrm{OCH}_{3}\right), 3.75\left(\mathrm{t}, 2 \mathrm{H}, \mathrm{S}-\mathrm{CH}_{2}\right), 4.15\left(\mathrm{t}, 2 \mathrm{H}, \mathrm{CH}_{2}-\mathrm{O}\right), 7.20-8.15(\mathrm{~m}, 6 \mathrm{H}, 5 \mathrm{H}$-aromatic $+1 \mathrm{H}, \mathrm{CH}-\mathrm{pyridine})$, 8.30 (s, $1 \mathrm{H}, \mathrm{NH}, \mathrm{D}_{2} \mathrm{O}$-exchangeable); ${ }^{13} \mathrm{C}-\mathrm{NMR}$ (DMSO-d6) $\delta: 12.30\left(\mathrm{CH}_{3}\right), 14.25\left(\mathrm{CH}_{3}\right), 30.85\left(\mathrm{CH}_{3}\right), 33.60\left(\mathrm{~S}-\mathrm{CH}_{2}\right)$, 50.72(C), 54.26(O-CH $\mathrm{CH}_{3}, \quad 63.8\left(\mathrm{CH}_{2}\right), \quad 104.12(\mathrm{C}), 117.81(2 \mathrm{CH}), 124.30(\mathrm{CH}), 125.0(\mathrm{CH}), 130.45(2 \mathrm{CH}), 135.16(\mathrm{CH})$, 137.77(C), 139.23(C), 150.24(C), 152.0(C-S), 155.71(C); Anal. Calcd. for $\mathrm{C}_{20} \mathrm{H}_{24} \mathrm{~N}_{6} \mathrm{OS}$ (396.51): C, 60.58; $\mathrm{H}, 6.10 ; \mathrm{N}$ 21.20 ; S, 8.09\%. Found: C, 60.93; H, 6.39; N, 21.42; S, 8.31\%. MS: $m / z$ 396.88( $\left(\mathrm{M}^{+}, 100\right)$

\section{5-(3,6-Dimethyl-1-phenyl-1H-pyrazolo[3,4-b]pyridine-5-yl)-2-(2-methoxy-ethyl)-5-methyl-[1,2,4]triazolidine-3-thione (15)}

A yellow crystals, $14.8 \%$, mp 192-194으. FT-IR (KBr) $\mathrm{V}_{\max } / \mathrm{cm}^{-1}: 3390$ and 3345 (N-H stretching), 3005 (C-H aromatic stretching), 2970 (C-H aliphatic stretching), 1600 (C=C stretching), 1215(C-O, stretching): ${ }^{1} \mathrm{H}$ NMR $\delta: 1.30\left(\mathrm{~s}, 3 \mathrm{H}, \mathrm{CH}_{3}\right), 2.2$ $(\mathrm{s}, 1 \mathrm{H}, \mathrm{NH}), 2.30(\mathrm{~s}, 1 \mathrm{H}, \mathrm{NH}), 2.55\left(\mathrm{~s}, 3 \mathrm{H}, \mathrm{CH}_{3}\right), 2.60\left(\mathrm{~s}, 3 \mathrm{H}, \mathrm{CH}_{3}\right), 3.85(\mathrm{~s}, 3 \mathrm{H}, \mathrm{OCH} 3), 4.60\left(\mathrm{t}, 2 \mathrm{H}, \mathrm{N}-\mathrm{CH}_{2}\right), 4.75(\mathrm{t}, 2 \mathrm{H}$, $\left.\mathrm{CH}_{2}-\mathrm{O}\right), 7.30-8.25\left(\mathrm{~m}, 6 \mathrm{H}, 5 \mathrm{H}\right.$-aromatic $+1 \mathrm{H}, \mathrm{CH}$-pyridine); ${ }^{13} \mathrm{C}-\mathrm{NMR}$ (DMSO-d6) $\delta: 11.78\left(\mathrm{CH}_{3}\right), 13.95\left(\mathrm{CH}_{3}\right), 30.48$ $\left(\mathrm{CH}_{3}\right), 43.34\left(\mathrm{O}-\mathrm{CH}_{3}\right), 46.27\left(\mathrm{~N}-\mathrm{CH}_{2}\right), 50.61(\mathrm{C}), 60.44\left(\mathrm{CH}_{2}\right), 105.23(\mathrm{C}), 115.71(2 \mathrm{CH}), 124.63(\mathrm{CH}), 125.11(\mathrm{CH}), 129.64$ $(2 \mathrm{CH}), 133.33(\mathrm{CH}), 137.81(\mathrm{C}), 139.90(\mathrm{C}), 151.11(\mathrm{C}), 152.47(\mathrm{C}), 172.0(\mathrm{C}=\mathrm{S})$; Anal. Calcd. for $\mathrm{C}_{20} \mathrm{H}_{24} \mathrm{~N}_{6} \mathrm{OS}(396.51)$ : C, $60.58 ; \mathrm{H}, 6.10 ; \mathrm{N}, 21.20 ; \mathrm{S}, 8.09 \%$. Found: C, 60.76; H, 6.42; N, 21.58; S, 8.28\%.

\section{2-[2-(3,6-Dimethyl-1-phenyl-1H-pyrazolo[3,4-b]pyridine-5-yl)-2-methyl-6-oxo-1,2,5,6-tetrahydro-[1,3]thiazolo[3,2- b][1,2,4]triazol-5-yl]-N-phenyl-acetamide (16)}

A mixture of $13(0.66 \mathrm{~g}, 0.002 \mathrm{~mol})$ and $\mathrm{N}$-phenylmaleimide $(0.35 \mathrm{~g}, 0.02 \mathrm{~mol})$ was refluxed for $2 \mathrm{~h}$ in glacial acetic (10 $\mathrm{mL}$ ). After cooling to r. t., the reaction mixture was poured into $50 \mathrm{~mL}$ of water. The precipitated colorless powder was filtered off, washed with methanol and recrystallized from ethanol to give compound 16 as yellow crystals, $65 \%$, mp 183 $185^{\circ} \mathrm{C}$. FT-IR $(\mathrm{KBr}) \mathrm{V}_{\max } / \mathrm{cm}^{-1}: 3395,3350(\mathrm{NH}), 3088(\mathrm{CH}-$ aromatic), $2987(\mathrm{CH}$ - aliphatic), $1710(\mathrm{C}=\mathrm{O}), 1690(\mathrm{C}=\mathrm{O})$, $1585(\mathrm{C}=\mathrm{N}) ;{ }^{1} \mathrm{H}$ NMR $\delta 1.40\left(\mathrm{~s}, 3 \mathrm{H}, \mathrm{CH}_{3}\right), 2.15(\mathrm{~s}, 1 \mathrm{H}, \mathrm{NH}), 2.55\left(\mathrm{~s}, 3 \mathrm{H}, \mathrm{CH}_{3}\right), 2.70\left(\mathrm{~s}, 3 \mathrm{H}, \mathrm{CH}_{3}\right), 2.80\left(\mathrm{dd}, J_{1}=3.66 \mathrm{~Hz}\right.$, $J_{2}=8.70,1 \mathrm{H}, \mathrm{CH}_{2}$ ), $3.30\left(\mathrm{dd}, J_{1}=4.35 \mathrm{~Hz}, J_{2}=1.85,1 \mathrm{H}, \mathrm{CH}_{2}\right), 4.60$ (dd, $J_{1}=3.50 \mathrm{~Hz}, J_{2}=0.98,1 \mathrm{H}, \mathrm{CH}-$ thiazole), $7.40-8.25$ $\left(\mathrm{m}, 11 \mathrm{H}, 10 \mathrm{H}\right.$-aromatic $+1 \mathrm{H}, \mathrm{CH}$-pyridine), $13.30(\mathrm{~s}, 1 \mathrm{H}, \mathrm{NH}) ;{ }^{13} \mathrm{C}-\mathrm{NMR}$ (DMSO-d6) $\delta: 12.55\left(\mathrm{CH}_{3}\right), 15.70\left(\mathrm{CH}_{3}\right)$, 29.11( $\left(\mathrm{CH}_{3}\right), 38.65\left(\mathrm{CH}_{2}\right), 48.45(\mathrm{CH}$ cyclic), 69.28(C), 106.23(C), $118.09(2 \mathrm{CH}), 120.35(2 \mathrm{CH}), 123.15(\mathrm{CH}), 124.98(\mathrm{CH})$, $128.66(2 \mathrm{CH}), 129.64(2 \mathrm{CH}), 130.45(\mathrm{C}), 137.07(\mathrm{C}), 140.33(\mathrm{C}), 141.72(\mathrm{C}), 150.51(\mathrm{C}), 152.18(\mathrm{C}), 159.36(\mathrm{C}), 164.11(\mathrm{C})$, 173.25( $\mathrm{C}=\mathrm{O}$ cyclic), $178.85(\mathrm{C}=\mathrm{O})$; Anal. Calcd. for $\mathrm{C}_{27} \mathrm{H}_{25} \mathrm{~N}_{7} \mathrm{O}_{2} \mathrm{~S}$ (511.60): C, 63.39; H, 4.93; N,19.16; S, 6.27. Found: C, 63.66; H, 5.16; N, 19.38; S, 6.60\%; MS: $m / z 511.39\left(\mathrm{M}^{+}, 73\right)$.

\section{3,6-Dimethyl-1-phenyl-5-(1,2,3-thiadiazol-4-yl)-1H-pyrazolo[3,4-b]pyridine (18)}

An excess amount of thionyl chloride $(10 \mathrm{~mL})$ was stirred at room temperature and the semicarbazone $17(0.97 \mathrm{~g}, 0.003$ $\mathrm{mol}$ ) was added in several portions. The mixtures were stirred at r.t. overnight until no more hydrogen chloride was produced, the product was washed with diethyl ether, dried, crystallized from benzene / pet.ether $40-60^{\circ} \mathrm{C}$ to afford brown powder of $18,83 \%, \mathrm{mp} 130-132^{\circ} \mathrm{C}$. FT-IR $(\mathrm{KBr}) \mathrm{V}_{\max } / \mathrm{cm}^{-1}: 3075(\mathrm{CH}-$ aromatic $), 2880(\mathrm{CH}-$ aliphatic $), 1590((\mathrm{C}=\mathrm{N})$, 1475((C=C); ${ }^{1} \mathrm{H}$ NMR $\delta 2.38\left(\mathrm{~s}, 3 \mathrm{H}, \mathrm{CH}_{3}\right), 2.55\left(\mathrm{~s}, 3 \mathrm{H}, \mathrm{CH}_{3}\right), 7.20-8.40(\mathrm{~m}, 6 \mathrm{H}, 5 \mathrm{H}$-aromatic $+1 \mathrm{H}, \mathrm{CH}-\mathrm{pyridine}), 9.75(\mathrm{~s}$, 
$1 \mathrm{H}, \mathrm{CH}$-thiadiazole); Anal. Calcd. for $\mathrm{C}_{16} \mathrm{H}_{13} \mathrm{~N}_{5} \mathrm{~S}$ (307.37): C, 62.52; $\mathrm{H}, 4.26 ; \mathrm{N}, 22.78 ; \mathrm{S}, 10.43$. Found: $\mathrm{C}, 62.81 ; \mathrm{H}, 4.57$; N, 23.08; S, 10.62\%; MS: $m / z 307.13\left(\mathrm{M}^{+}, 73\right)$.

\section{3,6-Dimethyl-1-phenyl-5-(1,2,3-selenadiazol-4-yl)-1H-pyrazolo[3,4-b]pyridine (19)}

The semicarbazone derivative $17(0.97 \mathrm{~g}, 0.003 \mathrm{~mol})$ was dissolved in glacial acetic acid $(25 \mathrm{~mL})$. To the hot solution, selenium dioxide powder $(0.55 \mathrm{~g}, 0.005 \mathrm{~mol})$ was added and the reaction mixture was stirred for $24 \mathrm{~h}$. The mixture was filtered and the filtrates poured into ice water and extracted with $\mathrm{CHCl}_{3}(3 \times 50 \mathrm{~mL})$. The combined organic layers were washed with a saturated sodium carbonate solution, dried over anhydrous sodium sulfate. After evaporation of the solvent the residue was crystallized from toluene to give brown powder of $19,74 \%, \mathrm{mp} 112-114^{\circ} \mathrm{C}$. $\mathrm{FT}-\mathrm{IR}(\mathrm{KBr}) \mathrm{V}_{\max } / \mathrm{cm}^{-1}$ : $3007\left(\mathrm{CH}\right.$ - aromatic), 2985(CH- aliphatic), $1595(\mathrm{C}=\mathrm{N}), 1480\left((\mathrm{C}=\mathrm{C}), 820(\mathrm{C}-\mathrm{Se}-\mathrm{N}) ;{ }^{1} \mathrm{H}\right.$ NMR $\delta 2.45(\mathrm{~s}, 3 \mathrm{H}, \mathrm{CH}), 2.61(\mathrm{~s}$, $\left.3 \mathrm{H}, \mathrm{CH}_{3}\right), 7.33-8.29(\mathrm{~m}, 6 \mathrm{H}, 5 \mathrm{H}$-aromatic $+1 \mathrm{H}, \mathrm{CH}$-pyridine $), 10.12(\mathrm{~s}, 1 \mathrm{H}, \mathrm{CH}$-selenadiazole); Anal. Calcd. for $\mathrm{C}_{16} \mathrm{H}_{13} \mathrm{~N}_{5} \mathrm{Se}$ (354.27): C, 54.24; H, 3.70; N,19.77\%. Found: C, 54.60; H, 3.92; N, 20.11; MS: m/z $354.19\left(\mathrm{M}^{+}, 56\right)$.

\section{3-Hydroxy-3-(2-(3,6-Dimethyl-1-phenyl-1H-pyrazolo[3,4-b]pyridine-5-yl)-2-oxoethyl)indolin-2-one (20).}

To a solid homogenous mixture of $1(0.52 \mathrm{~g}, 0.002 \mathrm{~mol})$ and isatine $(0.3 \mathrm{~g}, 0.002 \mathrm{~mol}), 10$ drops of dimethylamine was added and the mixture stirred for 15-30 minutes and a colorless solid formed was recrystallized from ethanol to give buff powder of 20, 61\%, mp 191-193으. FT-IR (KBr) $\mathrm{v}_{\max } / \mathrm{cm}^{-1}: 3500(\mathrm{OH}), 3410(\mathrm{NH}), 3080(\mathrm{CH}-$ aromatic), 2970(CH aliphatic), $1705(\mathrm{C}=\mathrm{O}), 1630(\mathrm{C}=\mathrm{O}) 1580(\mathrm{C}=\mathrm{N}), 1465(\mathrm{C}=\mathrm{C}) ;{ }^{1} \mathrm{H}$ NMR $\delta 2.20(\mathrm{~s}, 1 \mathrm{H}, \mathrm{OH}), 2.50(\mathrm{~s}, 3 \mathrm{H}, \mathrm{CH}), 2.70(\mathrm{~s}, 3 \mathrm{H}$, $\left.\mathrm{CH}_{3}\right), 3.15\left(\mathrm{~s}, 2 \mathrm{H}, \mathrm{CH}_{2}\right), 7.35-8.40(\mathrm{~m}, 10 \mathrm{H}, 9 \mathrm{H}$-aromatic $+1 \mathrm{H}, \mathrm{CH}$-pyridine), 8.60 (s, 1H, NH); Anal. Calcd. for $\mathrm{C}_{24} \mathrm{H}_{20} \mathrm{~N}_{4} \mathrm{O}_{3}$ (412.44): C, 69.89; H, 4.89; N,13.58\%. Found: C, 70.27; H, 4.95; N, 13.83.

\section{3-(2-(3,6-Dimethyl-1-phenyl-1H-pyrazolo[3,4-b]pyridine-5-yl)-2-oxoethylidene)indolin-2-one (21).}

A mixture of $20(0.4 \mathrm{~g}, 0.001 \mathrm{~mol})$, ethanol $25 \mathrm{~mL}$ and $40 \mathrm{~mL}$ of dilute $\mathrm{HCl}$ solution (25\%), was allowed to stand overnight, fine orange needles were formed and recrystallized from ethanol to give buff powder of $21,69 \%, \mathrm{mp} 210-212^{\circ} \mathrm{C}$. FT-IR $(\mathrm{KBr}) \mathrm{v}_{\max } / \mathrm{cm}^{-1}: 3395(\mathrm{NH}), 3087(\mathrm{CH}$-aromatic), 2990(CH- aliphatic), 1675(C=O), 1630(C=O), 1565(C=N ), 1480(C=C); H NMR $\delta 2.55\left(\mathrm{~s}, 3 \mathrm{H}, \mathrm{CH}_{3}\right), 2.68\left(\mathrm{~s}, 3 \mathrm{H}, \mathrm{CH}_{3}\right), 7.28-8.55(\mathrm{~m}, 11 \mathrm{H}, 10 \mathrm{H}$-aromatic $+1 \mathrm{H}, \mathrm{CH}$-pyridine), $8.70(\mathrm{~s}, 1 \mathrm{H}, \mathrm{NH})$; Anal. Calcd. for $\mathrm{C}_{24} \mathrm{H}_{18} \mathrm{~N}_{4} \mathrm{O}_{2}$ (394.43): C, 73.08; $\mathrm{H}, 4.60 ; \mathrm{N}, 14.20 \%$. Found: C, 73.31; $\mathrm{H}, 4.93 ; \mathrm{N}, 14.54 \%$.

\section{5-'(3,6-Dimethyl-1-phenyl-1H-pyrazolo[3,4-b]pyridine-5-yl)-2' , 4'-dihydrospiro[indol-3,3'-pyrazol]-2-(1H)-one (22).}

\section{Method A}

A mixture of $21(0.4 \mathrm{~g}, 0.001 \mathrm{~mol})$ and hydrazine hydrate $(0.20 \mathrm{~mL})$ in ethanol $(25 \mathrm{~mL})$ was heated under reflux for $4 \mathrm{~h}$, then left to cool, the residual material was filtered off and recrystallized from ethanol to afford yellow crystals of $22,71 \%$, $\mathrm{mp} 211-213^{\circ} \mathrm{C}$. FT-IR $(\mathrm{KBr}) \mathrm{V}_{\max } / \mathrm{cm}^{-1}: 3420,3365(\mathrm{NH}), 3069(\mathrm{CH}-$ aromatic $), 2877(\mathrm{CH}-$ aliphatic $), 1646(\mathrm{C}=\mathrm{O})$, $1570(\mathrm{C}=\mathrm{N}), 1473(\mathrm{C}=\mathrm{C}) ;{ }^{1} \mathrm{H}$ NMR $\delta 2.60\left(\mathrm{~s}, 3 \mathrm{H}, \mathrm{CH}_{3}\right), 2.70\left(\mathrm{~s}, 3 \mathrm{H}, \mathrm{CH}_{3}\right), 3.60\left(\mathrm{~s}, 2 \mathrm{H}, \mathrm{CH}_{2}\right), 6.30(\mathrm{~s}, 1 \mathrm{H}, \mathrm{NH}), 7.35-8.48$ $(\mathrm{m}, 10 \mathrm{H}, 9 \mathrm{H}$-aromatic $+1 \mathrm{H}, \mathrm{CH}$-pyridine), 8.63(s, $1 \mathrm{H}, \mathrm{NH}) ;$ Anal. Calcd. for $\mathrm{C}_{24} \mathrm{H}_{20} \mathrm{~N}_{6} \mathrm{O}(408.46): \mathrm{C}, 70.57 ; \mathrm{H}, 4.94 ;$ $\mathrm{N}, 20.58 \%$. Found: C, 70.84; $\mathrm{H}, 5.11$;, $20.79 \%$. MS: $\mathrm{m} / \mathrm{z} 408.30\left(\mathrm{M}^{+}, 82\right)$.

\section{Method B.}

To a mixture of $1(0.52 \mathrm{~g}, 0.002 \mathrm{~mol})$ and isatin $(0.3 \mathrm{~g}, 0.002 \mathrm{~mol})$ and, dimethylamine (5 drops) was added and the mixture was stirred for $30 \mathrm{~min}$ at room temperature. To the formed solid, glacial acetic acid (10 mL) and concentrated hydrochloric acid ( 3 drops) were added and the reaction mixture was heated at $80{ }^{\circ} \mathrm{C}$ for 30 min. Hydrazine hydrate $(0.02$ $\mathrm{mol}$ ) was added to the previous reaction mixture and heating was continued at $80 \stackrel{\circ}{\mathrm{C}}$ for $1 \mathrm{~h}$ to give spiro compound 22 . The product was identical with that formed by method $A$.

\section{5-'(3,6-Dimethyl-1-phenyl-1H-pyrazolo[3,4-b]pyridine-5-yl)-2' -phenyl-4'-dihydrospiro[indol-3,3'-pyrazol]-2-(1H)-one}

A mixture of $21(0.4 \mathrm{~g}, 0.001 \mathrm{~mol})$ and phenyl hydrazine $(0.5 \mathrm{~mL})$ in ethanol $(25 \mathrm{~mL})$ was heated under reflux for $7 \mathrm{~h}$, then left to cool, the residual material was filtered off and recrystallized from ethanol to afford yellow crystals of $23,63 \%$, $\mathrm{mp} 320-322^{\circ} \mathrm{C}$. FT-IR $(\mathrm{KBr}) \mathrm{V}_{\max } / \mathrm{cm}^{-1}: 3087\left(\mathrm{CH}\right.$-aromatic), 2889(CH- aliphatic), $1650(\mathrm{C}=\mathrm{O}), 1565(\mathrm{C}=\mathrm{N}), 1482(\mathrm{C}=\mathrm{C}) ;{ }^{1} \mathrm{H}$ NMR $\delta 2.64\left(\mathrm{~s}, 3 \mathrm{H}, \mathrm{CH}_{3}\right), 2.75\left(\mathrm{~s}, 3 \mathrm{H}, \mathrm{CH}_{3}\right), 3.49\left(\mathrm{~s}, 2 \mathrm{H}, \mathrm{CH}_{2}\right), 7.25-8.51(\mathrm{~m}, 15 \mathrm{H}, 14 \mathrm{H}$-aromatic $+1 \mathrm{H}, \mathrm{CH}-\mathrm{pyridine}), 8.60$ (s, $1 \mathrm{H}, \mathrm{NH}$ ); Anal. Calcd. for $\mathrm{C}_{30} \mathrm{H}_{24} \mathrm{~N}_{6} \mathrm{O}$ (484.55): C, 74.36; H, 4.99; N,17.34\%. Found: C, 70.55; H, 5.23; N, $17.70 \%$.

\section{3'-(3,6-Dimethyl-1-phenyl-1H-pyrazolo[3,4-b]pyridine-5-yl)- 4'-dihydrospiro[indol-3,5'-isoxazole]-2-(1H)-one (24).}

A mixture of compound $21(0.4 \mathrm{~g}, 0.001 \mathrm{~mol})$, hydroxylamine hydrochloride $(0.14 \mathrm{~g}, 0.002 \mathrm{~mol})$ and anhydrous sodium acetate $(0.085 \mathrm{~g}, 0.003 \mathrm{~mol})$ in absolute ethanol $(10 \mathrm{~mL})$ was refluxed for $6 \mathrm{~h}$. After cooling, the reaction mixture was poured onto ice-water $(50 \mathrm{~mL})$. The solid that formed was filtered off, air dried and recrystallized from absolute ethanol to afford white crystals of $24,66 \%, \mathrm{mp} 273-275^{\circ} \mathrm{C}$. FT-IR $(\mathrm{KBr}) \mathrm{v}_{\max } / \mathrm{cm}^{-1}: 3090(\mathrm{CH}-$ aromatic), $2880(\mathrm{CH}-$ aliphatic), 1655(C=O) , 1560(C=N), 1460(C=C) 1250 (C-O-N ); ${ }^{1} \mathrm{H}$ NMR $\delta 2.66$ (s, 3H, CH$\left.{ }_{3}\right), 2.79\left(\mathrm{~s}, 3 \mathrm{H}, \mathrm{CH}_{3}\right), 3.25\left(\mathrm{~s}, 2 \mathrm{H}, \mathrm{CH}_{2}\right)$, 7.25- $8.45\left(\mathrm{~m}, 10 \mathrm{H}, 9 \mathrm{H}\right.$-aromatic $+1 \mathrm{H}, \mathrm{CH}$-pyridine), $8.65(\mathrm{~s}, 1 \mathrm{H}, \mathrm{NH})$; Anal. Calcd. for $\mathrm{C}_{24} \mathrm{H}_{19} \mathrm{~N}_{5} \mathrm{O}_{2}(409.44): \mathrm{C}, 70.40 ; \mathrm{H}$, 4.68 ; N, $17.10 \%$. Found: C, $70.73 ; \mathrm{H}, 4.92 ; \mathrm{N}, 17.50 \%$. 
6'-(3,6-Dimethyl-1-phenyl-1H-pyrazolo[3,4-b]pyridine-5-yl)- spiro[indol-3,4' -pyrimidin-2'- (1H) thione]-2-(1H)-one (25).

A mixture of chalcone $21(0.4 \mathrm{~g}, 0.001 \mathrm{~mol})$, thiourea $(0.076 \mathrm{~g}, 0.001 \mathrm{~mol})$ and potassium hydroxide $(1.0 \mathrm{~g})$ in ethanol $(40$ $\mathrm{ml}$ ) was refluxed on a boiling water bath for one hour. The reaction mixture was left overnight and then concentrated under reduced pressure. The solid residue was collected, washed with water and recrystallized from ethanol to yield yellow crystals of $25,68 \%$, mp 172-174으. FT-IR $(\mathrm{KBr}) \mathrm{V}_{\max } / \mathrm{cm}^{-1}: 3340,3210,3180(\mathrm{NH}), 3085(\mathrm{CH}-$ aromatic $), 2890(\mathrm{CH}-$ aliphatic), 1645(C=O) , 1555(C=N), 1490(C=C) $1180(\mathrm{C}=\mathrm{S}) ;{ }^{1} \mathrm{H}$ NMR $\delta 2.55\left(\mathrm{~s}, 3 \mathrm{H}, \mathrm{CH}_{3}\right), 2.75\left(\mathrm{~s}, 3 \mathrm{H}, \mathrm{CH}_{3}\right), 6.20(\mathrm{~s}, 1 \mathrm{H}$, $\mathrm{CH}$-pyrimidine), 7.15- 8.35 (m, 10H, 9H-aromatic + 1H, CH-pyridine), 8.65(s, 1H, NH), 8.85 (s,br, $1 \mathrm{H}, \mathrm{NH}), 9.90(\mathrm{~s}, \mathrm{br}, 1 \mathrm{H}$, $\mathrm{NH}$ ); Anal. Calcd. for $\mathrm{C}_{25} \mathrm{H}_{20} \mathrm{~N}_{6} \mathrm{OS}$ (452.53): C, 66.35; H, 4.45; N,18.57; S, 7.09\%. Found: C, 66.59; H, 4.78; N, 18.88; S, $7.32 \%$.

\section{2-Cyano-N'-[1-(3,6-dimethyl-1-phenyl-1H-pyrazolo[3,4-b]pyridine-5-yl)-ethylidene]acetocarbohydrazide (26)}

To a solution of cyanoacetylhydrazine $(0.99 \mathrm{~g}, 0.001 \mathrm{~mol})$ in 1,4-dioxane $(20 \mathrm{~mL})$, compound 1 ( $0.26 \mathrm{~g}, 0.001 \mathrm{~mol})$ was added. The reaction mixture was heated under reflux for $4 \mathrm{~h}$ then poured onto a beaker containing an ice/water mixture. The formed solid product was collected by filtration and recrystallized from ethanol to give white crystals 0 f $26,68 \%$, mp 209-211 ${ }^{\circ} \mathrm{C}$. FT-IR $(\mathrm{KBr}) \mathrm{V}_{\max } / \mathrm{cm}^{-1}: 3365(\mathrm{NH}), 3090(\mathrm{CH}$-aromatic), 2880(CH-aliphatic), $2245(\mathrm{CN}), \quad 1679(\mathrm{C}=\mathrm{O})$, $1570(\mathrm{C}=\mathrm{N}), 1475(\mathrm{C}=\mathrm{C}) ;{ }^{1} \mathrm{H}$ NMR $\delta 1.90\left(\mathrm{~s}, 3 \mathrm{H}, \mathrm{CH}_{3}\right), 2.40\left(\mathrm{~s}, 3 \mathrm{H}, \mathrm{CH}_{3}\right), 2.78\left(\mathrm{~s}, 3 \mathrm{H}, \mathrm{CH}_{3}\right), 4.40\left(\mathrm{~s}, 2 \mathrm{H}, \mathrm{CH}_{2}\right), 7.26-8.30(\mathrm{~m}$, $6 \mathrm{H}, 5 \mathrm{H}$-aromatic $+1 \mathrm{H}, \mathrm{CH}$-pyridine); Anal. Calcd. for $\mathrm{C}_{19} \mathrm{H}_{18} \mathrm{~N}_{6} \mathrm{O}$ (346.39): C, 65.88; $\mathrm{H}, 5.24 ; \mathrm{N}, 24.26 \%$. Found: $\mathrm{C}, 66.13$; $\mathrm{H}, 5.49$; N, 24.60\%; ${ }^{13} \mathrm{C}-\mathrm{NMR}$ (DMSO-d6) $\delta: 11.95\left(\mathrm{CH}_{3}\right), 12.80\left(\mathrm{CH}_{3}\right), 15.60\left(\mathrm{CH}_{3}\right), 26.80\left(\mathrm{CH}_{2}\right), 105.79(\mathrm{C}), 113.64(\mathrm{CN})$, 118.50(2CH), 124.91(C), 126.11(C), 128.76(2CH), 132.81(C), 136.34(CH), 138.13(C), 150.41(C), 156.37(C), 158.22(C), $174.19(\mathrm{C}=\mathrm{O})$; MS: $\mathrm{m} / \mathrm{z} 346.54\left(\mathrm{M}^{+}, 62\right)$.

\section{3-(4-Chlorophenyl)-2-cyano-N'-[1-(3,6-dimethyl-1-phenyl-1H-pyrazolo[3,4-b]pyridine-5-yl)-ethylidene]acrylo- hydrazide (27)}

To a solution of $26(0.35 \mathrm{~g}, 0.001 \mathrm{~mol})$ in 1,4-dioxane $(20 \mathrm{~mL})$, p-chlorobenzaldehyde $(0.14 \mathrm{~g}, 0.001 \mathrm{~mol})$ was added. The reaction mixture was heated under reflux for $6 \mathrm{~h}$ then poured onto a beaker containing an ice/water mixture. The formed solid product was collected by filtration and and recrystallized from ethanol to give white crystals of $27,60 \%, \mathrm{mp} \mathrm{103-}$ 105으. FT-IR (KBr) $v_{\max } / \mathrm{cm}^{-1}: 3345(\mathrm{NH}), 3087(\mathrm{CH}-$ aromatic), 2969(CH- aliphatic), $2238(\mathrm{CN}), 1660(\mathrm{C}=\mathrm{O}), 1620(\mathrm{C}=\mathrm{N})$, 1498(C=C); ${ }^{1} \mathrm{H}$ NMR $\delta 1.65\left(\mathrm{~s}, 3 \mathrm{H}, \mathrm{CH}_{3}\right), 2.55\left(\mathrm{~s}, 3 \mathrm{H}, \mathrm{CH}_{3}\right), 2.65\left(\mathrm{~s}, 3 \mathrm{H}, \mathrm{CH}_{3}\right), 7.17-8.33(\mathrm{~m}, 11 \mathrm{H}, 9 \mathrm{H}$-aromatic $+1 \mathrm{H}, \mathrm{CH}-$ pyridine $+1 \mathrm{H}, \mathrm{C}=\mathrm{CH}$ ); Anal. Calcd. for $\mathrm{C}_{26} \mathrm{H}_{21} \mathrm{CIN}_{6} \mathrm{O}(468.94): \mathrm{C}, 66.59 ; \mathrm{H}, 4.51 ; \mathrm{Cl}, 7.56 ; \mathrm{N}, 17.92 \%$. Found: $\mathrm{C}, 66.81 ; \mathrm{H}$, $4.79 ; \mathrm{Cl}, 7.83 ; \mathrm{N}, 18.16 \%$.

\section{2-Oxo-N'-[1-(3,6-dimethyl-1-phenyl-1H-pyrazolo[3,4-b]pyridine-5-yl)-ethylidene]-2H-chromene-3-carbohydrazide} (28)

To a solution of $26(0.35 \mathrm{~g}, 0.001 \mathrm{~mol})$ in 1,4-dioxane $(20 \mathrm{~mL})$, salicylaldehyde $(0.12 \mathrm{~g}, 0.001$ mol) was added. The reaction mixture was heated under reflux for $5 \mathrm{~h}$ then poured onto a beaker containing an ice/water mixture. The formed solid product was collected by filtration and and recrystallized from ethanol to give white crystals of $28,64 \%, \mathrm{mp} 216$ 218으. FT-IR $(\mathrm{KBr}) \mathrm{v}_{\max } / \mathrm{cm}^{-1}: 3368(\mathrm{NH}), 3080(\mathrm{CH}$-aromatic), 2943(CH- aliphatic), $1670(\mathrm{C}=\mathrm{O}), 1650(\mathrm{C}=\mathrm{N}), 1490(\mathrm{C}=\mathrm{C})$; ${ }^{1} \mathrm{H}$ NMR $\delta 1.83\left(\mathrm{~s}, 3 \mathrm{H}, \mathrm{CH}_{3}\right), 2.50\left(\mathrm{~s}, 3 \mathrm{H}, \mathrm{CH}_{3}\right), 2.66\left(\mathrm{~s}, 3 \mathrm{H}, \mathrm{CH}_{3}\right), 7.20-8.35(\mathrm{~m}, 11 \mathrm{H}, 10 \mathrm{H}$-aromatic $+1 \mathrm{H}, \mathrm{CH}-\mathrm{pyridine})$, $8.72\left(\mathrm{~s}, 1 \mathrm{H}\right.$, CH-Coumarin); Anal. Calcd. for $\mathrm{C}_{26} \mathrm{H}_{21} \mathrm{~N}_{5} \mathrm{O}_{3}$ (451.48):C, 69.17; H, 4.69; N, 15.51\%. Found: $\mathrm{C}, 69.33$; $\mathrm{H}, 4.75$; N, $15.77 \%$.

\section{4-Amino-1-[1-(3,6-dimethyl-1-phenyl-1H-pyrazolo[3,4-b]pyridine-5-yl)-ethylideneamino]-2oxo-4-phenyl-1,2-dihydro pyridine-3,5-dicarbonitrile (29)}

To a solution of $26(0.35 \mathrm{~g}, 0.001 \mathrm{~mol})$ in 1,4-dioxane $(20 \mathrm{~mL})$, 2-benzylidenemalononitrile $(0.15 \mathrm{~g}, 0.001 \mathrm{~mol})$ was added. The reaction mixture was heated under reflux for $6 \mathrm{~h}$ then poured onto a beaker containing an ice/water mixture. The formed solid product was collected by filtration and and recrystallized from ethanol to give pale yellow crystals of $29,77 \%$, $\mathrm{mp} 324-326^{\circ} \mathrm{C}$. FT-IR $(\mathrm{KBr}) \mathrm{v}_{\max } / \mathrm{cm}^{-1}: 3452,3368\left(\mathrm{NH}_{2}\right), 3095(\mathrm{CH}-$ aromatic), 2971( $\mathrm{CH}$ - aliphatic),2229, $2189(2 \mathrm{CN})$, 1665(C=O), $1641(\mathrm{C}=\mathrm{N}), 1488(\mathrm{C}=\mathrm{C}) ;{ }^{1} \mathrm{H}$ NMR $\delta 2.11\left(\mathrm{~s}, 3 \mathrm{H}, \mathrm{CH}_{3}\right), 2.57\left(\mathrm{~s}, 3 \mathrm{H}, \mathrm{CH}_{3}\right), 2.64\left(\mathrm{~s}, 3 \mathrm{H}, \mathrm{CH}_{3}\right), 3.87\left(\mathrm{~s}, 2 \mathrm{H}, \mathrm{NH}_{2}\right)$, 7.27- $8.48\left(\mathrm{~m}, 11 \mathrm{H}, 10 \mathrm{H}\right.$-aromatic $+1 \mathrm{H}, \mathrm{CH}$-pyridine); Anal. Calcd. for $\mathrm{C}_{29} \mathrm{H}_{22} \mathrm{~N}_{8} \mathrm{O}$ (498.54):C, 69.87; $\mathrm{H}, 4.45 ; \mathrm{N}, 22.48 \%$. Found: C, $70.19 ; \mathrm{H}, 4.58 ; \mathrm{N}, 22.65 \%$.

\section{ANTIMICROBIAL ACTIVITY}

The antimicrobial activity of 22 selected compounds was screened against bacterial strains Staphylococcus aureus (AUMC B.54), Bacillus cereus (AUMC B.52) as gram-positive bacteria and Escherichia coli (AUMC B.53), Pseudomonas aeruginosa (AUMC B.73) as gram-negative bacteria and fungal strains Candida albicans (AUMC No.214), Aspergillus flavus (AUMC No.1276) using the agar well-diffusion method. ${ }^{31}$ all microbial strains were kindly provided by the Assiut University Mycological Centre (AUMC). To prepare inocula for bioassay, bacterial strains were individually cultured for $48 \mathrm{~h}$ in $100 \mathrm{~mL}$ conical flasks containing $30 \mathrm{~mL}$ nutrient broth medium. Fungi were grown for 7 days in $100 \mathrm{~mL}$ conicals containing $30 \mathrm{~mL}$ Sabouraud's dextrose broth. Bioassay was done in $10 \mathrm{~cm}$ sterile plastic Petri plates in which microbial suspension (1 mL/plate) and $15 \mathrm{~mL}$ appropriate agar medium (15 mL/plate) were poured. Nutrient agar and Sabouraud's dextrose agar were respectively used for bacteria and fungi. After solidification of the media, $5 \mathrm{~mm}$ diameter cavities were cut in the solidified agar (4 cavities/plate) using sterile cork borer. Chemical compounds dissolved in DMSO at $2 \% \mathrm{w} / \mathrm{v}(=20 \mathrm{mg} / \mathrm{mL})$ were pipetted in the cavities. The screening tests were carried 
out in triplicate and the results were expressed as a mean of three determinations. Chloramphenicol and Clotrimazole were used as standards. Data are represented as \% inhibition with reference to standards in (Table1).

\section{REFERENCES:}

[1] Mourad, Ch., Elena, S., Abdelouahid, S., and MarcoContelles, J. J. Heterocycl. Chem. 2010, 47, 861-872. Studies on the Acetylation of 3,6-Diamino-1H pyrazolo[3,4-b]pyridine-5-carbonitrile Derivatives.

[2] Parmar, S.S., Pandey. B.R., Dwivedi, C., and Ali. B. J. Med. Chem. 1974, 17, 1031-1033. 3-Hydroxy-1-nitrophenyl1(H-pyrazolo(3,4-b)pyridines as selective inhibitors of rat liver xanthine oxidase.

[3] Zhmurenko, L.A., Molodavkin, G.M., Voronina, T.A., and Lezina, V.P. J.Pharm. Chem. 2012, 46, 15-19. Synthesis and antidepressant and anxiolytic activity of derivatives of pyrazolo[4,3-c]pyridine and 4-phenylhydrazinonicotinic acids .

[4] Dias, L.R.S., Alvim. M.J., Freitas. A.C., Barreiro. E.J., and Miranda, A.L.P. Pharm. Acta. Helv.1994, 69, 163-169. Synthesis and analgesic properties of 5-acyl-arylhydrazone $1-\mathrm{H}$ pyrazolo [3,4-b] pyridine derivatives.

[5] Bare, T.M., McLaren, C.D., Campbell, J.B., Firor, J.W., Resch, J.F., Walters, C.P., Salama, A.I., Meiners, B.A., And Patel, J.B. J. Med. Chem. 1989, 32, 2561-2573.Synthesis and structure-activity relationships of a series of anxioselective pyrazolopyridine ester and amide anxiolytic agents.

[6] Falco, J.L., Lloveras, M., Buira, I., Teixido, J., and Borrell, J.I. Eur. J. Med. Chem . 2005, 40, 1179-1187. Design, synthesis and biological activity of acyl substituted 3-amino-5-methyl-1,4,5,7-tetrahydropyrazolo[3,4-b]pyridin-6-ones as potential hypnotic drugs.

[7] Bernardino, A.M.R., Azevedo, A.R., Pinheiro, L.C.S., Borges, J.C., Carvalho, V.L., Miranda, M.D., Meneses, M.D.F., Nascimento, M., Ferreira, D., Rebello, M.A., Silva, V.A.G., and Frugulhetti, I.C. Med. Chem. Res. 2007, 16, 352-369. Synthesis and Antiviral activity of new 4-(phenylamino)/4-[(methylpyridin-2-yl)amino]-1-phenyl-1 $\mathrm{H}$ - pyrazolo[3,4b]pyridine-4-carboxylic acids derivatives.

[8] Tucker, T.J., Sisko, J.T., Tynebor, R.M., Williams, T.M., Felock, P.J., Flynn, J.A., Lai, M.T., Liang, Y., McGaughey, G., Liu, M., Miller, M., Moyer, G., Munshi, V., Perlow-Poehnelt, R., Prasad, S., Reid, J.C., Sanchez, R., Torrent, M., Vacca, J.P., Wan, B.L., and Yan, Y. J. Med. Chem . 2008, 51, 6503-6511. Discovery of 3-\{5-[(6-Amino-1H-pyrazolo[3,4b]pyridine-3-yl)methoxy]-2-chlorophenoxy\}-5-chlorobenzonitrile (MK-4965): A Potent, Orally Bioavailable HIV-1 NonNucleoside Reverse Transcriptase Inhibitor with Improved Potency against Key Mutant Viruses.

[9] Stasch, J.P., Dembowsky, K., Perzborn, E., Stahl, E., and Schramm, M.B. J. Pharmacol. 2002, 135, 344-355. Cardiovascular actions of a novel NO independent guanylyl cyclase stimulator, BAY 41-8543: in vivo studies.

[10] Dyck, B., Grigoriadis, D.E., Gross, R.S., Guo, Z., Marinkovic, D., McCarthy, J.R., Moorjani, M., Regan, C.F., Saunders, J., Schwaebe, M.K., Szabo, T., Williams, J.P., Zhang, X., Bozigian, H., and Chen, T.K. J. Med. Chem. 2005, 48,4100-4110. Potent, Orally Active Corticotropin-Releasing Factor Receptor-1 Antagonists Containing a Tricyclic Pyrrolopyridine or Pyrazolopyridine Core.

[11] Hoehn, H., Polacek, I., and Schulze, E. J. Med. Chem. 1973, 16, 1340-1346. Potential antidiabetic agents. Pyrazolo[3,4-b]pyridines.

[12] Kuczynski, L., Mrozikiewicz, A., Banaszkiewicz, W., and Poreba, K. Pol. J. Pharmacol.Pharm. 1979, 31, $217-225$. Synthesis and biological activity of pyrazo-[3,4-b]-pyridine derivatives. Part I.

[13] Abdel-Aziz, H.A., Saleh, T.S., and El-Zahabi, H.A.S. Arch. Pharm. 2010, 343, 24-30. Facile Synthesis and In-Vitro Antitumor Activity of Some Pyrazolo[3,4-b]pyridines and Pyrazolo[1,5-a]pyrimidines Linked to a Thiazolo[3,2 a]benzimidazole Moiety.

[14] Cunico, W., Cechinel, C.A., Bonacorso, H.G., Martins, M.A., Zanatta, N., de Souza, M.V., Freitas, I.O., Soares, R.P., and Krettli, A.U. Bioorg. Med. Chem. Lett. 2006, 16, 649-653. Antimalarial activity of 4-(5-trifluoromethyl-1 H pyrazol-1yl)-chloroquine analogues

[15] El-Emary, T.I., Kamal El-Dean, A.M., and El-Kashef, H.S. II Farmaco, 1998, 53, 383-388. Facile synthesis of some new pyrazolo [3,4-b] pyrazines and their antifungal activity.

[16] El-Kashef, H.S., El-Emary, T.I., Gasquet, M., Timon-David, P., Maldonado, J., and Vanelle, P. Pharmazie, 2000, 55, 572-576. New pyrazolo[3,4-b]pyrazines: Synthesis and biological activity.

[17] El-Emary, T.I., and Abdel-Mohsen, S.A. Molecules, 2012, 17, 14464-14483. Multi-Component One-Pot Synthesis and Antimicrobial Activities of 3-Methyl- 1,4-diphenyl-7-thioxo-4,6,8,9-tetrahydropyrazolo[5,4-b]pyrimidino[5,4e]pyridine-5-one and Related Derivatives.

[18] Bernardino, A.M.R., Rolim, A.M., and Castro, H.C. Bioorg. Med. Chem . 2006, 14, 5765-5770. Design, synthesis, SAR, and biological evaluation of new (phenylamino)pyrazolo[2,3-b]pyridine4- derivatives".

[19] Wust, F., Kniess, T., Kretzschmar, M., and Bergmann, R. Bioorg. Med. Chem. Lett. 2005, 15,1303-1306. Synthesis and biodistribution of a 18F-labelled corticosteroid as ligand for mapping brain glucocorticoid receptors by means of PET. 
[20] Helosia, D.M., Aurea, E., Alice, M.B., Marilene, C.C., and Leonor, L.L. J. Med. Chem. 2004, 47, 5427-5432. Antileishmanial Pyrazolopyridine Derivatives:Synthesis and Structure-Activity Relationship Analysis.

[21] Yu, G., Mason, H.J., Wu, X., Wang, J., Chong, S., Dorough, G., Henwood, A., Pongrac, R., Seliger, L., He, B., Normandin, D., Adam, L., Krupinski, J., and Macor, J.E. J. Med. Chem. 2001, 44, 1025-1027. Substituted pyrazolopyridines as potent and selective PDE5 inhibitors: potential agents for treatment of erectile dysfunction.

[22] El-Kashef, H.S. and El-Emary, T.I. J. Chem. Res. Synop. 1998, 20-21. Polycyclic Pyrazoles: Routes to New Pyrazoloazines.

[23] El-Emary, T.I., and Bakhite, E.A. Pharmazie, 1999, 54, 106-111. Synthesis and biological screening of new 1,3diphenylpyrazoles with different heterocyclic moieties at position-4.

[24] El-Emary, T.I., Al-Muaikel, N., and Moustafa, O.S. Phosphorus Sulfur Silicon Relat. Elem.2002, 177, $195-210$. Synthesis and Antimicrobial Activity of some New Heterocycles Based on 3-Methyl-1-Phenyl-5-Benzene Sulfonamido Pyrazole.

[25] El-Emary, T.I., Khalil, A., El-Hag, G.A., and El-Adasy, M. A. Phosphorus Sulfur Silicon Relat. Elem. 2005, 180, $19-30$. A Facile Synthesis of Some New Thiazolo[3, 2]pyridines Containing Pyrazolyl Moiety and Their Antimicrobial Activity.

[26] Haeufel ,J., and Breitmeir, E. Angew. Chem. Int. Ed. Engl. 1974, 13: 604-609. Synthesis of Pyrazolo Heteroaromatic Compounds by Means of 5-Amino-3-methyl-1-phenylpyrazole-4-carbaldehyde.

[27] El-Emary, T.I. j. Chin. Chem. Soc.2007, 54, 507-518. Synthesis of Newly Substituted Pyrazoles and Substituted Pyrazolo[3,4-b]pyridines Based on 5-Amino-3-methyl-1-phenylpyrazole.

[28] Haythem, A.S., Ibrahim, M. M., Amal, G.A., and Mohammad, S.M. Monatsh. Chem. 2010, 141,471-478.Synthesis and antimicrobial activity of new 1,2,4-triazole-3-thiol metronidazole derivatives.

[29] Saravanan, S., Nithya, A., and Muthusubramanian, S. J. heterocycl. Chem. 2006, 43, 149-155. Synthesis and characterization of 4-aryl-5-(1-aryl-2-methyl-2-nitropropyl)-1,2,3-selenadiazoles .

[30] Sondhi, S.M., Dinodia, M., and Kumar, .A. Bioorg. Med. Chem. 2006, 14,4657-4663. Synthesis, anti-inflammatory and analgesic activity evaluation of Some amidine and hydrazone derivatives.

[31] Kwon-Chung, K.J., and Bennett, J.W. Med. Mycol. Lea. Febiger. Philadel. 1992, 81-102. Principles of antifungal and antibacterial therapy . 